U.S.N.A. --- Trident Scholar project report; no. 272 (2000)

\title{
DETERMINING THE MOTIONS OF NEAR EARTH ASTEROIDS THROUGH OPTICAL OBSERVATIONS
}

\author{
by \\ Midshipman Douglas T. Campbell, Class of 2000 \\ United States Naval Academy \\ Annapolis, Maryland

(signature)
Certification of Adviser Approval
$\begin{gathered}\text { Assistant Professor Debora M. Katz-Stone } \\ \text { Department of Physics }\end{gathered}$
$\frac{\text { (signature) }}{\text { (date) }}$

DISTRIBUTION STATEMENT A

Approved for Public Release

Distribution Unlimited

Acceptance for the Trident Scholar Committee

Professor Joyce E. Shade

Chair, Trident Scholar Committee

(signature)

(date) 
USNA-1531-2 


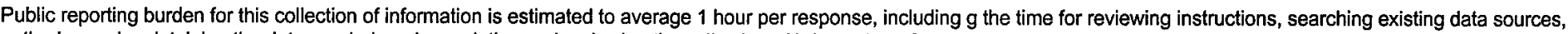

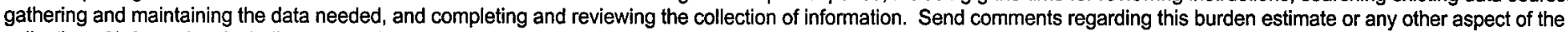

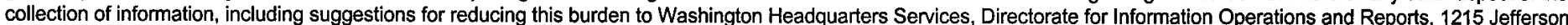
Davis Highway, Suite 1204, Arlington, VA 22202-4302, and to the Office of Management and Budget, Paperwork Reduction Project (0704-0188), Washington, DC 20503.

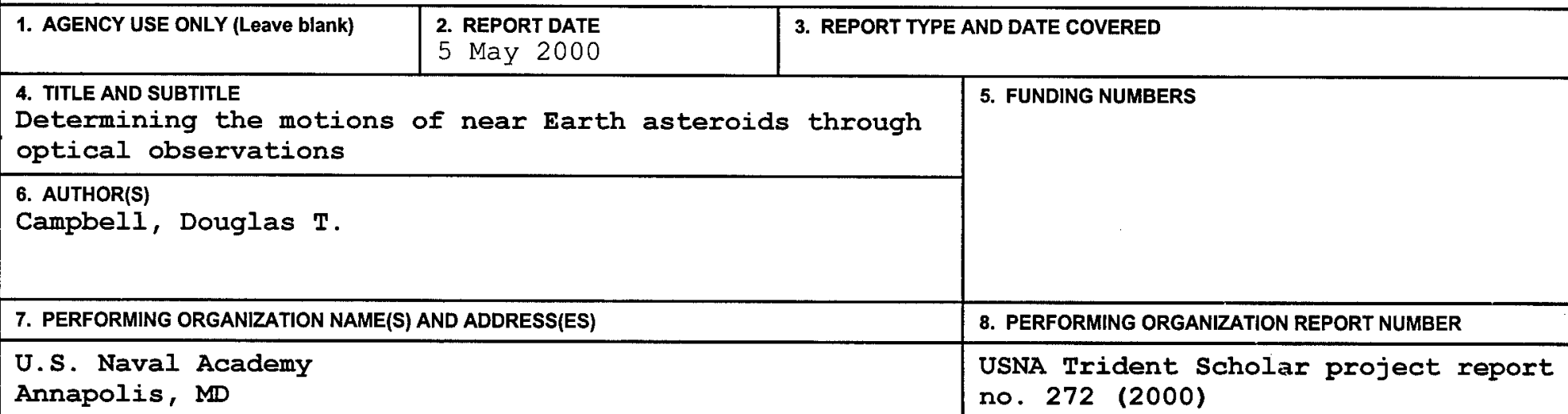

Annapolis, MD no. 272 (2000)

9. SPONSORING/MONITORING AGENCY NAME(S) AND ADDRESS(ES)

10. SPONSORING/MONITORING AGENCY REPORT NUMBER

11. SUPPLEMENTARY NOTES

Accepted by the U.S. Trident Scholar Committee

12a. DISTRIBUTION/AVAILABILITY STATEMENT

This document has been approved for public release; its distribution

12b. DISTRIBUTION CODE

is UNLIMITED.

13. ABSTRACT:. An Earth impact with an asteroid has potentially devastating consequences. In order to avoid global destruction, astronomers have been attempting to map out the orbits of all possibly hazardous Near-Earth Asteroids (NEAs) for the past two decades. Although there are a number of astronomers devoted to discovering new NEAs, there are very few who are tracking these discoveries. Therefore many recently identified NEAs are routinely lost. This Trident Scholar Project had two main objectives. The scientific goal of this project focused upon tracking several NEAs in order to determine their orbits. In addition, lightcurves were observed for a subset of these NEAs in order to find their rotational periods and their general shapes. This was done by taking observations of each asteroid for at least one full night and then plotting light intensity versus time. The second objective was to refurbish and upgrade the observatory at the U.S. Naval Academy. Astronomical research at the U.S. Naval Academy hinges on the successful operation of a 20" Cassegrain telescope with a Photometrics 1024x1024 Charge Coupled Device (camera), as well as several computers to control these devices and process the data. As a result of this Trident Scholar Project, the U.S. Naval Academy now boasts a fully functioning, state-of-the-art observatory, including newly acquired and developed software for tracking NEAs and analyzing astronomical observations in general. This Trident Scholar Project added to the body of knowledge of NEAs by determining the orbits of eleven asteroids, as well as the rotational periods and rough shapes of four asteroids. In addition, this Trident Scholar Project will benefit both midshipmen and faculty who wish to either continue tracking NEAs or begin a new field of astronomical research using the now completely established observatory at the U.S. Naval Academy.

\section{SUBJECT TERMS \\ NEA, Near-Earth Asteroid, Asteroid, Telescope, Charge-Coupled Device, CCD, Orbit, Lightcurve, Astronomy}

15. NUMBER OF PAGES

16. PRICE CODE

\begin{tabular}{|l|l|l|l|}
\hline $\begin{array}{l}\text { 17. SECURITY CLASSIFICATION } \\
\text { OF REPORT }\end{array}$ & $\begin{array}{l}\text { 18. SECURITY CLASSIFICATION } \\
\text { OF THIS PAGE }\end{array}$ & $\begin{array}{l}\text { 19. SECURITY CLASSIFICATION } \\
\text { OF ABSTRACT }\end{array}$ & 20. LIMITATION OF ABSTRACT \\
\hline
\end{tabular}




\section{ABSTRACT}

An Earth impact with an asteroid has potentially devastating consequences. In order to avoid global destruction, astronomers have been attempting to map out the orbits of all possibly hazardous Near-Earth Asteroids (NEAs) for the past two decades.

Although there are a number of astronomers devoted to discovering new NEAs, there are very few who are tracking these discoveries. Therefore many recently identified NEAs are routinely lost.

This Trident Scholar Project had two main objectives. The scientific goal of this project focused upon tracking several NEAs in order to determine their orbits. In addition, lightcurves were observed for a subset of these NEAs in order to find their rotational periods and their general shapes. This was done by taking observations of each asteroid for at least one full night and then plotting light intensity versus time.

The second objective was to refurbish and upgrade the observatory at the U.S. Naval Academy. Astronomical research at the U.S. Naval Academy hinges on the successful operation of a 20" Cassegrain telescope with a Photometrics 1024x1024 Charge Coupled Device (camera), as well as several computers to control these devices and process the data. As a result of this Trident Scholar Project, the U.S. Naval Academy now boasts a fully functioning, state-of-the-art observatory, including newly acquired and developed software for tracking NEAs and analyzing astronomical observations in general.

This Trident Scholar Project added to the body of knowledge of NEAs by determining the orbits of eleven asteroids, as well as the rotational periods and rough shapes of four asteroids. In addition, this Trident Scholar Project will benefit both 
midshipmen and faculty who wish to either continue tracking NEAs or begin a new field of astronomical research using the now completely established observatory at the U.S. Naval Academy.

KEYWORDS: NEA, Near-Earth Asteroid, Asteroid, Telescope, Charge-Coupled Device, CCD, Orbit, Lightcurve, Astronomy 


\section{ACKNOWLEDGEMENTS}

I would like to thank Assoc. Prof. Katz-Stone for all of her help, guidance, and long nights throughout this project. I would also like to thank Dr. Jeffery Larsen at Spacewatch for his help in understanding how to do this project. I would like to thank Prof. Albert for the advice and help she has given me to get the observatory up and running. I would like to thank Jure Skvarc for his assistance with Fitsblink. I would also like to thank Dave Moore, Dave Rector, and Jeff Walbert for helping me take care of the telescope. I would like to thank Ian Huss and Mark Kelly for their help with fixing the telescope's many problems. I would finally like to thank the Trident Scholar Committee, Dean Miller, and the United States Naval Academy Physics Department for allowing me to do this project. 


\section{TABLE OF CONTENTS}

ABSTRACT 1

ACKNOWLEDGEMENTS 3

1 INTRODUCTION 5

2 OBSERVATIONS 8

2.1 Equipment $\quad 8$

2.2 Determining General Instrument Parameters 12

2.3 A Typical Night's Observation $\quad 15$

2.4 Calibration and Data Reduction $\quad 17$

$\begin{array}{ll}2.5 \text { Identifying NEAs } & 18\end{array}$

3 ORBITS 20

3.1 Gauss' Method 22

3.2 Determination of Orbital Elements $\quad 27$

3.3 Perturbed Motion $\quad 29$

4 LIGHTCURVES $\quad 30$

5 RESULTS AND DISCUSSION

5.1 Cooperation Process 32

5.2 Using an Astronomical Calibrator $\quad 35$

5.3 Orbital Results and Discussion $\quad 36$

5.4 Lightcurve Results and Discussion $\quad 49$

6 CONCLUSION

7 FUTURE WORK $\quad 56$

\begin{tabular}{ll} 
REFERENCES & 57 \\
\hline
\end{tabular}

APPENDIX A: LAPLACE'S METHOD $\quad 58$ 


\section{$\underline{1 .}$ Introduction}

In 1993, Drs. Carolyn and Eugene Shoemaker and amateur astronomer David Levy made an astonishing discovery. They found a fragmented comet, later named Shoemaker-Levy 9, caught in Jupiter's gravitational field. Over the course of about a year, astronomers around the world watched as the comet further split apart into more than twenty fragments and then as each fragment struck Jupiter. The impacts ranged from barely discernable to the equivalent of about six million megatons of TNT. If comet Shoemaker-Levy 9 had hit Earth, it is unlikely there would be any human life remaining (e.g., Zelik \& Gregory 1998; Boslough \& Crawford 1995 and references therein).

In fact, it is likely that such an impact destroyed the dinosaurs. Luis and Walter Alvarez studied an impact crater on the Yucatan Peninsula (Alvarez et al. 1980). They found a layer of iridium in the Cretaceous-Tertiary boundary, which corresponds to the epoch of dinosaur extinction. Iridium is not normally found on the Earth's crust because it is believed to have sunk to the center of the Earth during its formation; on the other hand, asteroids are often rich in iridium. Therefore, the Alvarezes believe that the iridium must have come from a large asteroid or comet (Alvarez et al. 1980; Alvarez 1990), and this impact led to the extinction of the dinosaurs. Studies of these two powerful impacts have awoken an increasing sense of urgency in the scientific community and in the U.S. Congress. Accordingly, a new goal has been set to map the orbit of all 2000 potentially devastating near-earth objects.

The size of the asteroid determines how destructive it will be upon impact. An asteroid that is $10 \mathrm{~km}$ in diameter will cause earthquakes of magnitude 12 on the Richter 
Scale $^{1}$ with amplitudes of $200 \mathrm{~m}$. The energy from this sort of impact will raise the atmosphere's temperature to about $500^{\circ} \mathrm{F}$. Large chunks of rock would be thrown up in the air. Many would escape the pull of the Earth's gravity. The rest would fall back to Earth, crashing into the entire planet. An asteroid of about $1 \mathrm{~km}$ in diameter could destroy a continental area directly, and it would cause a nuclear winter due to the dust thrown up into the atmosphere. The dust would block the Sun's radiation, cooling the Earth to temperatures far too cold for humans. An asteroid of about $0.1 \mathrm{~km}$ would not annihilate the entire world population, but it would cause a great amount of damage. If it hits land, an asteroid of this size could easily destroy an area the size of a state. If a 0.1 $\mathrm{km}$ asteroid hits water, which is very likely because the surface area of the Earth is $3 / 4$ water, it would create a tsunami that would drown an entire coastline. Finally, a $10 \mathrm{~m}$ asteroid poses no threat to humanity as a whole. However, the crater, debris, and fires caused by its impact would still cause much damage (see, e.g. Arny 1998; Toon et al. 1994).

In order to focus upon preventing global destruction, it is imperative to find and track all Near-Earth Asteroids (NEAs) that are at least $1 \mathrm{~km}$ in diameter. This worldwide effort is coordinated by the Minor Planet Center $^{2}$ which collects data on each newly discovered asteroid and disseminates the necessary information via the world-wide web

\footnotetext{
${ }^{1}$ The Richter Scale is a logarithmic scale, devised in 1935 to identify the magnitude of an earthquake. The value calculated reflects the amount of energy released. The Richter scale ranges from 0 to 8.9 , the highest recorded quake. Because it is a logarithmic scale, an earthquake of 6.0 is 10 times more severe than one of 5.0 .

${ }^{2}$ The Minor Planet Center (MPC) operates at the Smithsonian Astrophysical Observatory, under the auspices of Commission 20 of the International Astronomical Union (IAU) and is a nonprofit organization, with principal funding coming from subscriptions to the various services offered by the Center. The MPC is responsible for the efficient collection, computation, checking and dissemination of astrometric observations and orbits for minor planets and comets, via the Minor Planet Circulars (issued monthly) and the Minor Planet Electronic Circulars.
} 
to other astronomers who do the follow-up tracking of these newly discovered asteroids. Only a handful of observatories ${ }^{3}$ are dedicated to discovering new asteroids and even fewer professional observatories track asteroids. In fact, the majority of NEAs discovered are lost. The major goals of this Trident Project were to develop a program here at USNA to track NEAs and to observe several NEAs in order to determine their orbits, rotational periods, and basic shapes.

\footnotetext{
${ }^{3}$ In the United States this effort is led by Spacewatch in Arizona, LINEAR in New Mexico and NEAT in Hawaii.
} 


\section{Observations}

\section{$\underline{2.1 \text { Equipment }}$}

The telescope used for this project is a Cassegrain reflecting telescope manufactured by DFM Engineering, Inc. ${ }^{4}$ and mounted on the roof of Michelson Hall (Figure 1). The telescope has a 20" parabolic primary mirror, which collects the light from astronomical sources and focuses it on a convex secondary mirror in the middle of the telescope. This secondary mirror then reflects the light through a hole in the center of the parabolic mirror and focuses it on the detector (e.g., Shu 1982). In addition, an IBM computer controls the movements of the telescope and dome. In particular, the dome and telescope must spin to compensate precisely for the Earth's rotation rate. This allows observations of a single object for up to an hour without resetting the telescope's pointing position.

A good astronomical detector is a device that produces a predictable response to the light gathered and focused by the telescope. Early astronomers used the human eye as their detector. This was later replaced by photographic film and photomultipliers. Today, modern astronomers use charge coupled devices (CCDs), which were first dèveloped in the early 1980 's. While their costs may be prohibitive for some observers, their advantages over other detectors are crucial for many projects. First, CCDs, unlike photographic film, have a linear response rate $^{5}$ over a large range of intensities. Second, CCDs are very sensitive, detecting about $50 \%$ of the incident photons. In addition, they

\footnotetext{
${ }^{4}$ DFM Engineering, Inc. Longmont, Colorado

${ }^{5}$ The CCD's response is directly proportional to the number of incident photons.
} 


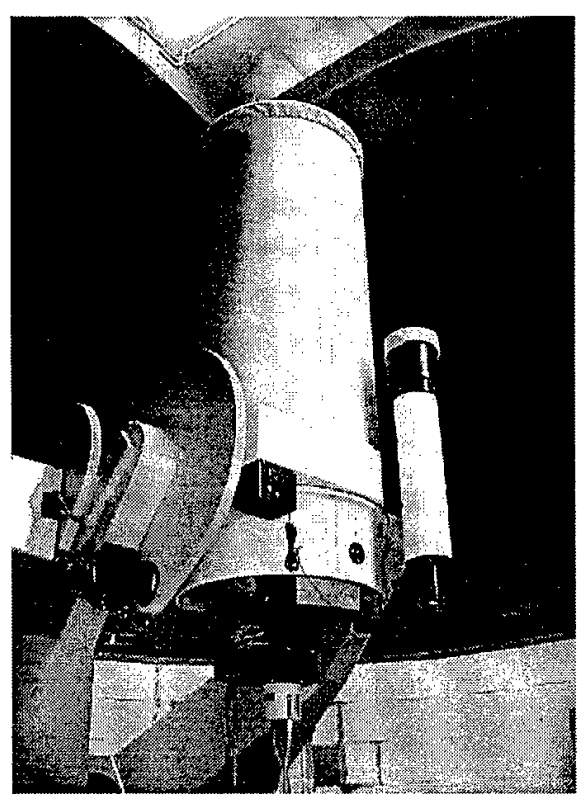

Fig. 1.-- This DFM telescope was used for all the observations taken at USNA. The small telescope is the finder scope.
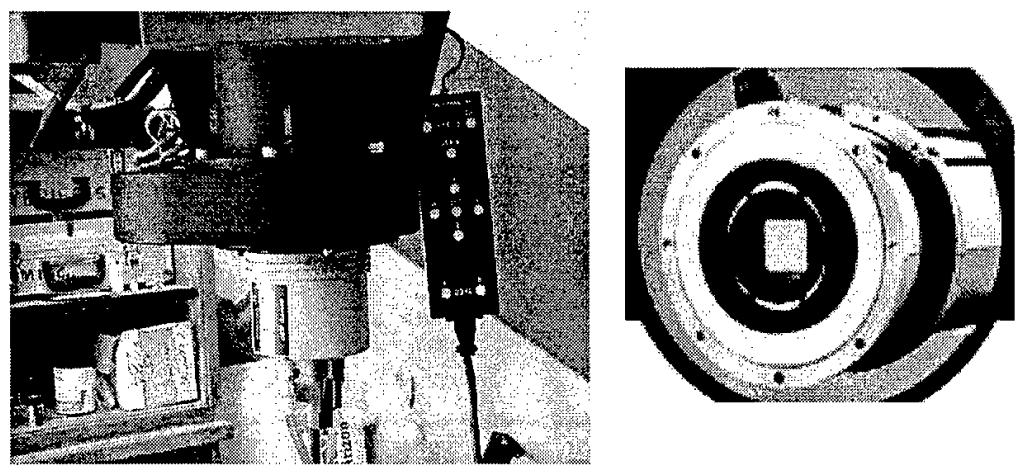

Fig. 2. -- Left: A close-up of the Photometrics CCD used to image all the observations taken at USNA. Right: The interior of the CCD. The small square near the center of the image is the location of the actual CCD chip. 
are high resolution detectors with micron-sized pixels. Another advantage is that, with a CCD, the contamination caused by nearby stars or cosmic rays can be removed from the image. A final advantage is that the readout of a CCD is already digitized and easily fed directly into a computer, which is used to calibrate and analyze the data.

The detector used for this project is a CCD manufactured by Photometrics ${ }^{6}$ (Figure 2). Like all CCDs, it is made up of photosensors, which can be imagined as little squares on a chessboard but so small that millions can fit in a few square centimeters. Our CCD has 1024 by 1024 photosensors. Each photosensor is made of a light sensitive material which, when struck by a photon, releases an electron. Each pixel is held at a positive potential so that the electrons are trapped in the potential well during the exposure. The CCD is controlled by a Micron computer, which is also used to archive and calibrate the data.

After the exposure is completed, the computer reads the potential off each pixel one at a time. The $\mathrm{CCD}$ does this with a horizontal register, which has as many pixels as a line in the array (1024) but is covered so as not to be exposed to the light. In order to read the potential off of the $\mathrm{CCD}$, vertical clocks transfer each line in a CCD to the one directly below it, and horizontal clocks transfer the pixel's electric charge laterally down the horizontal register to the register's extremity for the output, as shown in Figure 3. This procedure is repeated until all of the pixels have been read (e.g., Martinez \& Klotz 1999). Once this is done, the computer converts the potential of each pixel into counts and assembles a picture.

\footnotetext{
${ }^{6}$ Photometrics, Roper Scientific Inc. Tucson, Arizona.
} 
Fig. 3. -- This illustrates how a single pixel is read off the CCD. This process repeats until all pixels have been read.
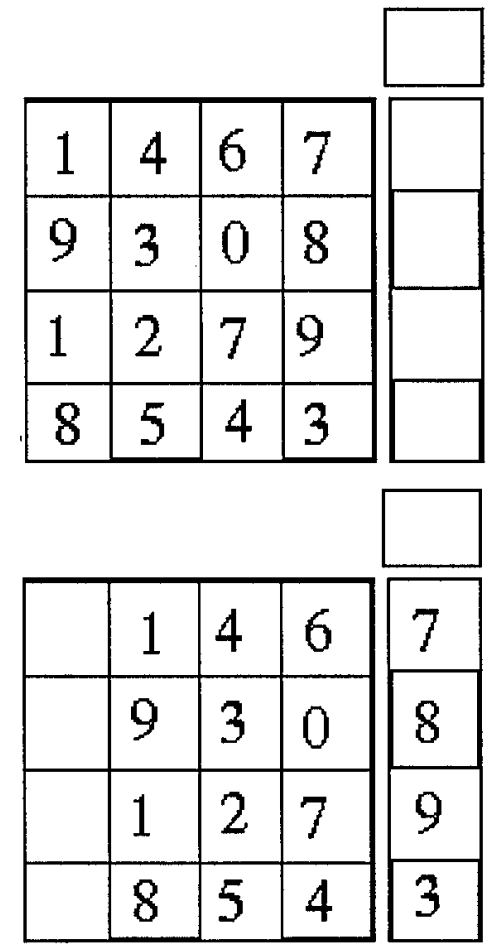

7

\begin{tabular}{|l|l|l|l|l|}
\hline & 1 & 4 & 6 & 8 \\
\hline & 9 & 3 & 0 & 9 \\
\hline 1 & 2 & 7 & 3 \\
\hline & 8 & 5 & 4 & \\
\hline
\end{tabular}

The CCD is exposed to light and a charge pattern forms.

The charge is shifted one column. The first column is shifted into the serial register.

The charge in the serial register is shifted up. The first pixel is shifted into the output node where it is subsequently collected for processing. 
Unfortunately, the CCD is not only sensitive to the light from the secondary mirror, but also to thermal radiation produced by the electronics of the CCD itself, known as the "dark" current. To reduce the dark current, antifreeze is used to chill the CCD to approximately $-40^{\circ} \mathrm{C}$. This is further complicated because the dark current increases the longer a pixel is exposed to the thermal radiation. So as each row of the CCD is transferred to the horizontal register, it is still exposed to the dark current. The first row read has a smaller dark current than the last row read. Therefore, all images have a gradient across them.

Finally, the sensitivity of each pixel varies. The response factor for the pixels can be affected by the gain irregularities of the CCD's pixels and from problems with optical transmission, such as vignetting ${ }^{7}$ and the presence of dust in the light path (Martinez and Klotz 1999).

\subsection{Determining General Instrument Parameters}

Before observing any asteroids, some basic information about the equipment was needed. Between the telescope and detector is an eight-filter wheel. Each filter allows only one waveband of light through.

The focal point of the optical system is different for each filter. The focus for each filter is determined by trial and error. For each filter, a series of pictures of a star field were observed at different focuses. The image in which the stars appear point-like rather than donut-like corresponds to the right focus for that filter. In order to choose the best filter for observing these faint NEAs, NGC 7790, a known star cluster was observed 
using all eight of the filters as well as without a filter. NGC 7790, as shown in Figure 4 , is a cluster of stars whose magnitudes, image, and scale has been previously observed and published (Odewahn et al. 1992). The best images of NGC 7790 were observed with a Kodak filter that removes any light bluer than $5900 \AA^{8}$. This filter removes artificial light produced by sodium and mercury lamps.

Next, the resolution and field of view of the instrument needed to be determined. This was done by observing the same standard star field, NGC 7790. The magnitudes of the stars and distances between the stars on the plane of the sky are known. The resolution is determined by taking a number of measurements of the distance between stars on both the exposure, Figure 5, in pixels and the published picture of NGC 7790 in $\operatorname{arcseconds}^{9}$. Comparing these two measurements gives the pixel size in arcseconds. By taking several measurements, they can be averaged to obtain a reliable value of 0.885 arcsec/pixel. The diffraction ${ }^{10}$ limited resolution of a 20 " telescope is 0.23 arcsec. Since this is so much smaller than the pixel size, the pixel size effectively becomes the resolution. The field of view is then just 1024 pixels $\times 0.885 \mathrm{arcsec} / \mathrm{pixel}=906 \mathrm{arcsecs}$. This is about half of the angular size of the moon.

Finally, this same standard field was used to determine the exposure time required to observe objects as faint as a typical NEA, about $18^{\text {th }}$ magnitude ${ }^{11}$. To see the faintest object in NGC 7790, about 18.5 in magnitude, a one-minute exposure was needed.

\footnotetext{
${ }^{7}$ Light loss or "fall-off" from the center of the lens to the outside edge due to the lens being slightly smaller than the array.

${ }^{8} 1 \AA=10^{-10} \mathrm{~m}$

${ }^{9} 1$ arcsec $=1 / 3600$ degrees

${ }^{10}$ Diffraction describes the bending of light as it passes through an aperture.

${ }^{11}$ A unitless number, based on a logarithmic scale, used to describe the brightness of an astronomical object. Faint objects have larger magnitudes than bright objects
} 


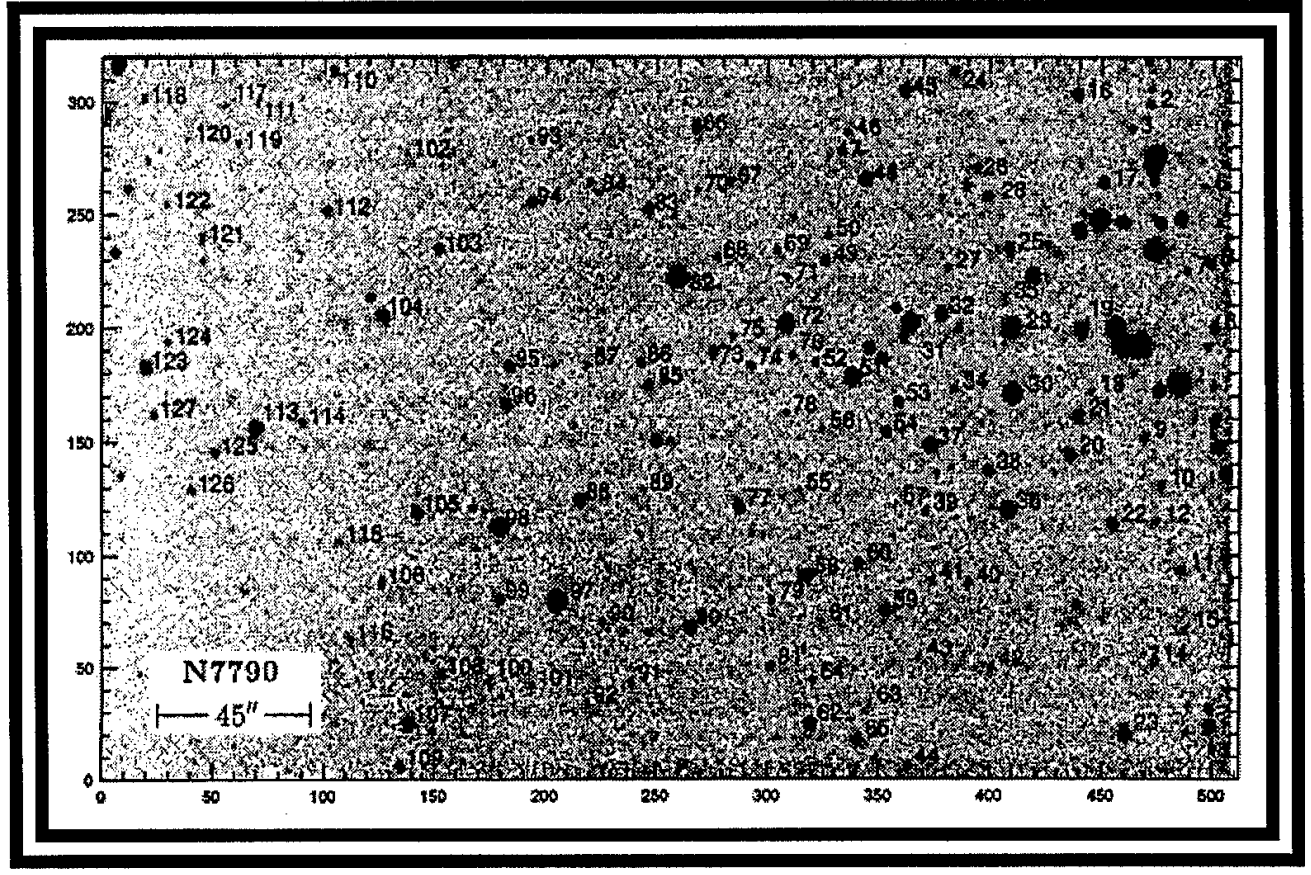

Fig. 4. -- This star field (NGC 7790) was observed by Odewahn et al. (1992). It was used to determine basic parameters for optical system.

$\mathrm{m}_{1}-\mathrm{m}_{2}=2.5 \log \mathrm{f}_{2} / \mathrm{f}_{1}$

where $f_{i}$ is the flux (power/area) of object $i$ and $m_{i}$ is its magnitude. 


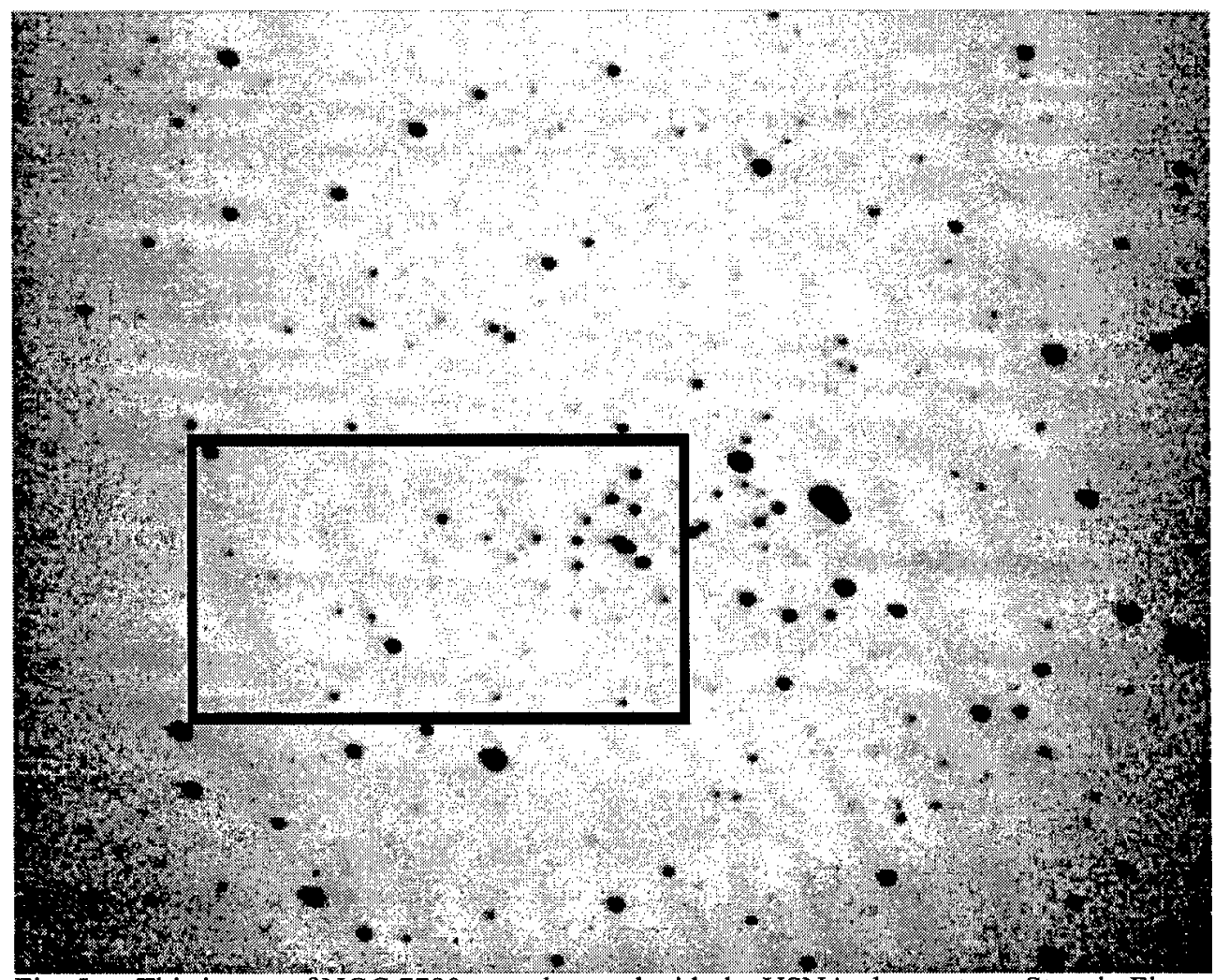

Fig. 5. -- This image of NGC 7790 was observed with the USNA observatory. Stars in Figure 4 and the boxed region were compared to determine basic parameters as explained in the text.

2.3 A Typical Night's Observation

A typical night's observation begins in the late afternoon when the CCD and chiller are turned on so that the $\mathrm{CCD}$ has enough time to be cooled to an operating temperature of $-40^{\circ} \mathrm{C}$. Then, just before dusk, flat-field images are taken which will be needed later to calibrate the data. Flat-field images are used to correct for differences in the response of the photosensors. To reduce the dark current in these images, the exposure time is short, less than five seconds. To increase the signal-to-noise ratio (SNR) in the final flat-field image and to reduce the effects of the transient events such as cosmic rays, twenty to forty flat-field images are taken, and the median of these images is found. Flat-field images should be uniformly illuminated and free of stars. Therefore, as the sun sets, the telescope is moved to the west to avoid imaging stars. 
About a half-hour before observing an asteroid, the telescope's alignment needs to be calibrated. The telescope is manually pointed at a known object, and a picture is taken. Usually, the image is not centered. The telescope is manually adjusted until the object is at the center of the CCD. Now, the telescope's computer is reinitialized. This is a difficult process because the CCD's field of view is too small to identify constellations and individual stars look alike. In order to overcome this difficulty, the telescope is first pointed at a planet such as Jupiter or Saturn, a very distinguishable object. Once the planet is centered and the computer is reinitialized, the telescope is close to being aligned. To refine the alignment, the computer is used to point the telescope to a bright star such as Vega. A short exposure is taken and only Vega appears near the center of the CCD, because the alignment is close and Vega is so bright. Now, the telescope is manually moved until Vega is in the center of the CCD and the computer is reinitialized one last time. That aligns the telescope accurately.

Finally, an NEA can be observed. The Minor Planet Center maintains a list of newly discovered asteroids on-line. It also provides software, which extrapolates an asteroid's ephemeris. ${ }^{12}$ The telescope is pointed at the asteroid and an exposure is taken. In a single image, an asteroid looks exactly like a faint star. In order to distinguish an asteroid from a star at least two images must be taken; in principle, the object that moved between exposures is the asteroid. The asteroid must move fifty pixels before another exposure can be taken so that the asteroid has moved far enough for the human eye to easily distinguish the change in position. The time between exposures is determined from the estimated speed of the asteroid. While only two exposures are required to detect

\footnotetext{
${ }^{12}$ An ephemeris is the estimated position of an object as a function of time based on previous observations.
} 
an asteroid, at least three exposures are taken for each asteroid in case there is a problem with one of the images and to help properly identify the asteroid.

Before the end of the night, two more series of images, which are used to calibrate the data, must be taken. As described in Section 2.1, each photosensor has an initial positive potential. To measure this pre-charge potential, a series of "bias" images is taken. These are short $(1 \mathrm{~ms})$ exposures taken with the shutter closed. The other series of images are used to measure the dark current. The dark current depends on both the temperature of the CCD and the exposure time. Therefore, the series of dark images must have the same exposure time that was used during the asteroid observations and with the CCD at the same temperature.

\subsection{Calibration and Data Reduction}

As explained in Sections 2.1 and 2.3, a raw image is contaminated by the dark current and pre-charge potential and has a gain, which varies from pixel to pixel. Calibration is the process that corrects for these known effects. In a raw image, the intensity of any pixel $(x, y)$ is given by

$$
I(x, y)=b(x, y)+d(x, y, t, T)+i(x, y) r(x, y)
$$

where $I(x, y)$ is the raw image, $b(x, y)$ is the bias value, $d(x, y, t, T)$ is the dark value, $r(x, y)$ is the response factor, and $i(x, y)$ is the final calibrated image for an exposure time $t$ at temperature T (e.g., Martinez and Klotz 1999). Solving for the calibrated image, gives

$$
i(x, y)=\frac{I(x, y)-(b(x, y)+d(x, y, t, T))}{r(x, y)}
$$


The bias value is estimated by taking a series of very short exposure, with the shutter closed. Since the exposure time is short, the dark current built up during the exposure can be ignored. To increase the SNR in the bias image, the median of a series of ten bias images is found,

$$
\mathrm{l}_{\mathrm{b}}(\mathrm{x}, \mathrm{y})=\mathrm{b}(\mathrm{x}, \mathrm{y})
$$

where $l_{b}$ is the median bias image. A median is taken, not a mean, because the normal bias value for each pixel is being determined. However, transient events such as cosmic rays affect the bias value of a random pixel. By taking a median, this abnormality is ignored. If a mean is taken, however, a cosmic ray would dominate the average bias value of that pixel and thus gives the incorrect bias value.

The dark value is found by taking an image with the shutter closed at the same temperature and for the same exposure time as the image. As with the bias images, the median is found from a series of ten images. However this median image $\left(l_{d}\right)$ also contains the bias value, so

$$
l_{d}(x, y)=b(x, y)+d(x, y, t, T) .
$$

Finally, the response factor is found by taking a series of flat-field images (as described in Section 2.3) and finding their median. The flat-field image still contains the bias value, but has an exposure time of one to five seconds so that the dark current can be ignored.

$$
\mathrm{l}_{\mathrm{f}}(\mathrm{x}, \mathrm{y})=\mathrm{b}(\mathrm{x}, \mathrm{y})+\mathrm{r}(\mathrm{x}, \mathrm{y})^{*} \mathrm{C}
$$

The normalization constant, $\mathrm{C}$ is the average of the median flat-field image. Substituting equations (3), (4), and (5) into (2) gives the formula for the calibrated image, 


$$
i(x, y)=\frac{I(x, y)-1_{d}(x, y)}{1_{f}(x, y)-1_{b}(x, y)} C
$$

\subsection{Identifying NEAs}

Once the calibrated image is determined, the next step is identifying the asteroid using an analysis program known as Fitsblink ${ }^{13}$ running on a Dell Computer. First, all of the real sources in the image are determined. The parameters must be chosen carefully so that all the real sources are identified without picking up false detections. For example, real sources tend to be at least two pixels in extent and have at least 900 counts. NEAs, unlike stars, move rapidly across the sky. Therefore the easiest way to distinguish an asteroid from a faint star is to observe its motion. This is done by taking two or more images. As explained above, the time separation of the two images should be such that the asteroid moves fifty pixels in that time. Then, the two images are registered on top of one another by aligning the same bright stars found in both images. Next, the two images are "blinked", which means the computer display rapidly switches between the two images. Since the images are aligned, the object that moves must be the asteroid. Once the asteroid has been detected, the stars in the field need to be matched to stars in the Guide Star Catalog ${ }^{14}$ or the U.S. Naval Observatory Catalog ${ }^{15}$ in order to get the position and velocity (astrometry) as well as the magnitude (photometry) of the asteroid. Once

\footnotetext{
${ }^{13}$ Fitsblink is a program written by Jure Skvarc that was used to do astrometry and photometry. It aligns two images over one another and blinks them to identify the asteroid. It also compares and matches an observer's image to an image from the Guide Star Catalog so that the coordinates of every pixel are determined.

${ }^{14}$ The guide star index is provided by the Hubble Space Telescope Science Institute. It was constructed from 1477 Schmidt plates; it contains entries for over 18 million objects between 8.5 and $15^{\text {th }}$ magnitude. ${ }^{15}$ The USNO SA2.0 guide star index was put together by the U.S. Naval Observatory. It contains over 50 million stars observed in both the blue and red Palomar Optical Sky Survey.
} 
the astrometry is completed, a new ephemeris is calculated so that the next observational position is determined, and these data will be used in the final calculation of the asteroid's orbit. 


\section{Orbits}

Near-Earth Asteroids (the focus of this project) are split into three categories: Amors, Apollos, and Atens. Amor asteroids have orbits that are inside the orbit of Mars. While Amors never cross Earth's orbit, they may still travel close to Earth's orbit. Apollo asteroids are sometimes called Earth-crossing asteroids because their aphelion ${ }^{16}$ is beyond Earth's orbit while their perihelion ${ }^{17}$ is inside the Earth's orbit. Aten asteroids have an orbit with a semi-major axis close to $1 \mathrm{AU}^{18}$, so they spend most of their time in the Earth's orbital path (e.g., Fix 1999).

A single observation of an asteroid consists of determining its position in the plane of the sky, not its distance along our line of sight, at the time of observation. In order to determine its orbit, a minimum of three observations is required. With three observations, the asteroid's heliocentric position $\left(\mathbf{r}_{i}\right)$, velocity $\left(\dot{\mathbf{r}}_{i}\right)$ and acceleration $\left(\ddot{\mathbf{r}}_{i}\right)$ as a function of time are estimated. An orbit may be calculated from these estimates.

A fully determined orbit is described mathematically by six orbital elements: the longitude of the ascending node ${ }^{19}(\Omega)$, the angle of inclination $(i)$ with the ecliptic ${ }^{20}$ plane, the argument of perihelion ( $(\omega)$, the semi-major axis (a), the eccentricity (e), and the mean anomaly $\left(\mathrm{M}_{0}\right)$. The longitude of the ascending node $(\Omega)$ is the angle measured

\footnotetext{
${ }^{16}$ An object's farthest point from the Sun.

${ }^{17}$ An object's closest point to the Sun.

${ }^{18}$ An Astronomical Unit, $\mathrm{AU}$, is the distance from Earth to the Sun. $1 \mathrm{AU} \approx 93$ million miles

${ }^{19}$ The ascending node is the point in the orbit that crosses the ecliptic from South to North.

${ }^{20}$ The ecliptic is the plane of Earth's orbit and is traced out by the apparent path of the Sun during the year.
} 
eastward from the vernal equinox ${ }^{21}$ to the ascending node. The inclination angle $(i)$ is the angle between the ecliptic and the plane of orbit such that $0 \leq i<180^{\circ}$. The argument of perihelion $(\omega)$ is the angle measured in the direction of the orbital motion, from the ascending node to the direction of the perihelion. These relationships can be seen graphically in Figure 6 (e.g., Danby et al. 1995). The semi-major axis (a) is half the length of the major axis of an ellipse. The eccentricity (e) of a closed orbit is a parameter between 0 and 1 such that a circle has an eccentricity of 0 . The mean anomaly $\left(\mathrm{M}_{0}\right)$ specifies the position of the object at a fiducial time.

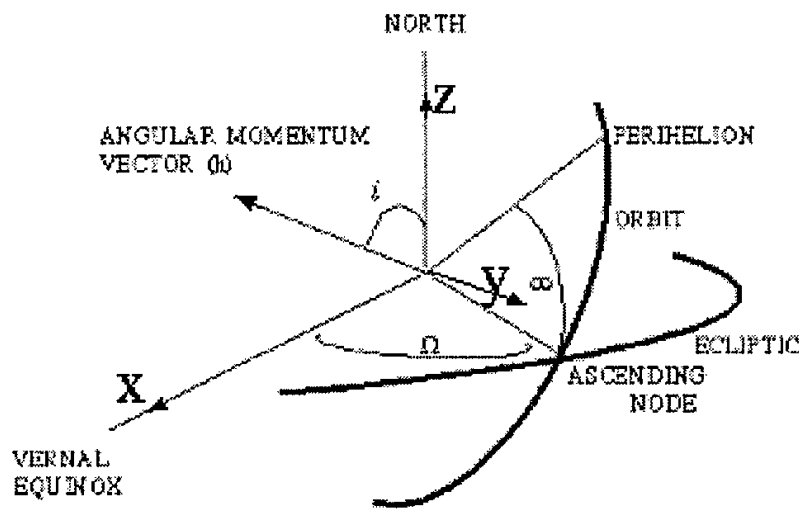

Fig. 6.--Elements of an orbit.

There are two classical techniques for determining these orbital elements:

Laplace's Method and Gauss' Method. Laplace's Method directly solves the equation of motion described by the gravitational interactions in the solar system. In practice, this method is difficult because it requires numerical differentiation of a minimal amount of data. Like the Spacewatch observatory, this Trident Project uses Gauss' Method. Gauss'

\footnotetext{
${ }^{21}$ The vernal equinox is the ascending node on the celestial sphere where the celestial equator crosses the ecliptic.
} 
Method does not use the equations of motion at one instant of time. Instead, it uses the solution of the unperturbed two-body problem to bridge the time interval between observations. This method requires Taylor Series expansions as explained below (Taff 1984). Following Danby (1988), Gauss' Method is reviewed below while Laplace's Method reviewed in Appendix A.

\subsection{Gauss' Method}

Gauss' Method was developed two hundred years ago and since then has been continually improved upon. It involves taking three observations, bridging them with a Taylor Series expansion and solving for the position $\mathbf{r}_{\mathrm{i}}$ of the object at the three times. Once $\mathbf{r}_{\mathbf{i}}$ is found, the orbital elements can be determined.

As shown in Figure 7, the positions at the three times, $\mathbf{r}_{1}, \mathbf{r}_{2}$, and $\mathbf{r}_{3}$ all lie in a plane and $\mathbf{r}_{2}$ can be expressed in terms of $\mathbf{r}_{1}$ and $\mathbf{r}_{3}$,

$$
\mathbf{r}_{2}=c_{1} \mathbf{r}_{1}+c_{3} \mathbf{r}_{3}
$$

where $c_{1}$ and $c_{2}$ are constants.
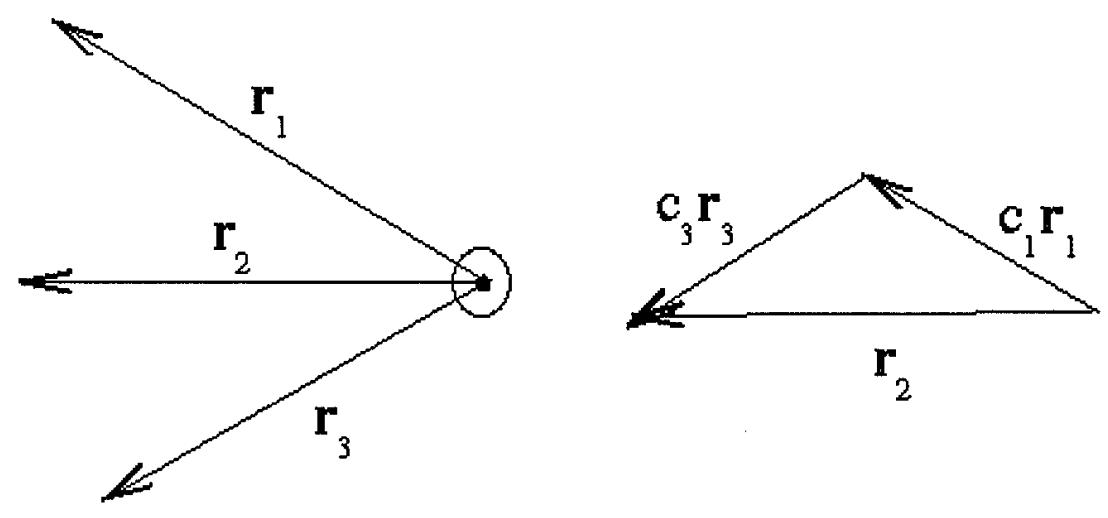

Fig. 7.-- Geometrical representation of equation (7). 
The position $\mathbf{r}(\mathrm{t})$ can be written as a Taylor series, which can be reduced to an expression in terms of a fiducial position and velocity ( $\mathbf{r}_{0}$ and $\left.\dot{\mathbf{r}}_{0}\right)$,

$$
r(t)=f(t) r_{0}+g(t) \dot{r}_{0}
$$

where $\mathbf{r}_{0}$ and $\dot{\mathbf{r}}_{0}$ are the position and velocity vectors at time $t_{0}$, and $f$ and $g$ are series,

$$
\begin{aligned}
& f(t)=1-\frac{1}{2} \sigma\left(t-t_{0}\right)^{2}+\frac{1}{2} \sigma \beta\left(t-t_{0}\right)^{3}+\ldots \\
& g(t)=\left(t-t_{0}\right)-\frac{1}{6} \sigma\left(t-t_{0}\right)^{3}+\ldots
\end{aligned}
$$

where

$$
\sigma=\frac{\mathrm{GM}_{\text {sun }}}{\mathrm{r}_{0}^{3}} \equiv \frac{\mu}{\mathrm{r}_{0}^{3}}, \quad \text { and } \quad \beta=\frac{\mathbf{r}_{0} \bullet \dot{\mathbf{r}}_{0}}{\mathrm{r}_{0}^{2}}
$$

Therefore, the positions at $t_{1}$ and $t_{3}$ are written in terms of $\mathbf{r}_{2}$ and $\dot{\mathbf{r}}_{2}$ as

$$
\begin{aligned}
& \mathbf{r}_{1}=\mathrm{f}_{1} \mathbf{r}_{2}+\mathrm{g}_{1} \dot{\mathbf{r}}_{2} \\
& \mathbf{r}_{3}=\mathrm{f}_{3} \mathbf{r}_{2}+\mathrm{g}_{3} \dot{\mathbf{r}}_{2},
\end{aligned}
$$

and

$$
\mathbf{r}_{2}=c_{1}\left(f_{1} \mathbf{r}_{2}+g_{1} \dot{\mathbf{r}}_{2}\right)+c_{3}\left(f_{3} \mathbf{r}_{2}+g_{3} \dot{\mathbf{r}}_{2}\right)
$$

The next step is finding $c_{1}$ and $c_{3}$ by eliminating $\mathbf{r}_{\mathrm{i}}$ and $\dot{\mathbf{r}}_{\mathrm{i}}$. Taking the cross products of $\dot{\mathbf{r}}_{2}$ and $\mathbf{r}_{2}$ with equation (12) yields

$$
1=c_{1} f_{1}+c_{3} f_{3} \text { and } 0=c_{1} g_{1}+c_{3} g_{3} \text {. }
$$

Solving equation (13) for $c_{1}$ and $c_{2}$ gives

$$
\begin{aligned}
& c_{1}=\frac{g_{3}}{f_{1} g_{3}-g_{1} f_{3}} \\
& c_{3}=-\frac{g_{1}}{f_{1} g_{3}-g_{1} f_{3}} .
\end{aligned}
$$


The geometrical association between the Sun, Earth, and the asteroid can be seen in Figure 8. Since $\mathbf{r}$ is neither known nor even observed, the observer's relation to the asteroid must be introduced, $\rho$. From the geometry shown in Figure 8,

$$
\mathbf{R}+\mathbf{r}=\boldsymbol{\rho}=\rho \hat{\boldsymbol{\rho}} .
$$

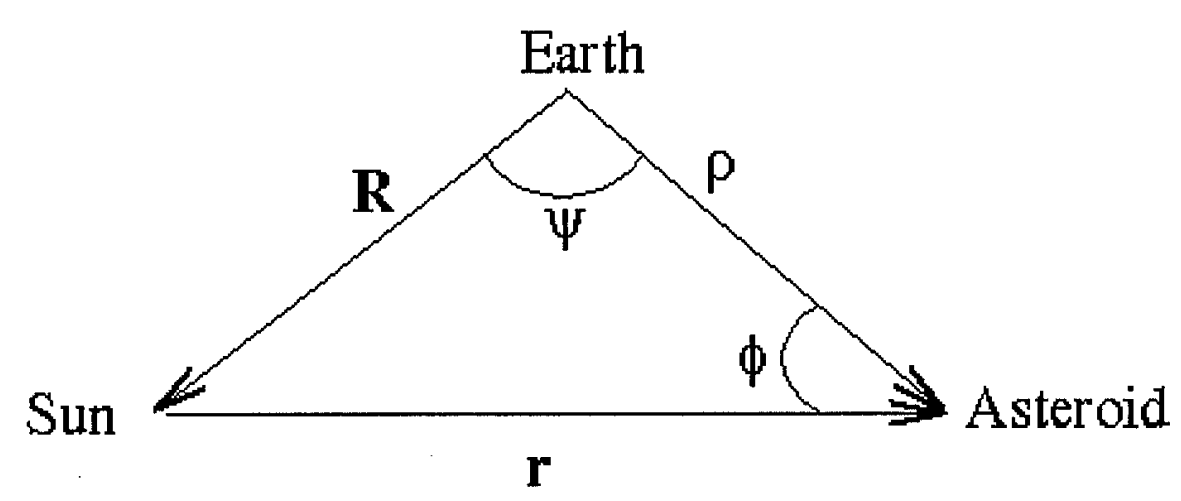

Fig. 8. -- Geometrical representation of equation (15).

Substituting this into equation (7),

$$
c_{1} \rho_{1} \hat{\boldsymbol{\rho}}_{1}-\rho_{2} \hat{\boldsymbol{\rho}}_{2}+\mathrm{c}_{3} \rho_{3} \hat{\boldsymbol{\rho}}_{3}=\mathrm{c}_{1} \mathbf{R}_{1}-\mathbf{R}_{2}+\mathrm{c}_{3} \mathbf{R}_{3}
$$

and substituting in equation (11)

$$
\begin{aligned}
\mathrm{f}_{1} \mathbf{r}_{2}+\mathrm{g}_{1} \dot{\mathbf{r}}_{2} & =\rho_{1} \hat{\boldsymbol{\rho}}_{1}-\mathbf{R}_{1} \\
\mathbf{r}_{2} & =\rho_{2} \hat{\boldsymbol{\rho}}_{2}-\mathbf{R}_{2} \\
\mathrm{f}_{3} \mathbf{r}_{2}+\mathrm{g}_{3} \dot{\mathbf{r}}_{2} & =\rho_{3} \hat{\boldsymbol{\rho}}_{3}-\mathbf{R}_{3} .
\end{aligned}
$$

In order to solve for $\mathbf{r}_{2}$, it is convenient to change coordinate systems from a heliocentric system to a geocentric system. The new coordinate system uses the axes $\xi$, $\eta$, and $\zeta$, where the $\xi$-axis is pointing towards the first observed position, the direction of 
the third observation intersects the positive $\eta$-axis, and $\zeta$ is mutually perpendicular to both $\xi$ and $\eta$ as seen in Figure 9.

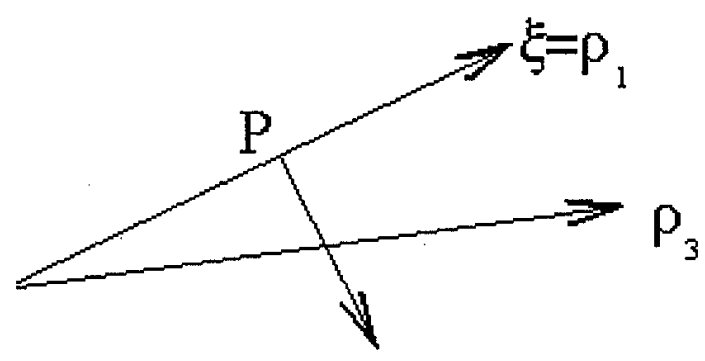

$\eta$

Fig. 9. -- This illustrates the coordinate transformation.

In this case,

$$
\begin{aligned}
& \hat{\boldsymbol{\eta}}=\frac{\hat{\boldsymbol{\rho}}_{1} \times\left(\hat{\boldsymbol{\rho}}_{3} \times \hat{\boldsymbol{\rho}}_{1}\right)}{\left|\hat{\boldsymbol{\rho}}_{1} \times\left(\hat{\boldsymbol{\rho}}_{3} \times \hat{\boldsymbol{p}}_{1}\right)\right|} \\
& =\frac{\hat{\boldsymbol{\rho}}_{3}\left(\hat{\boldsymbol{\rho}}_{1} \bullet \hat{\boldsymbol{\rho}}_{1}\right)-\hat{\boldsymbol{\rho}}_{1}\left(\hat{\boldsymbol{\rho}}_{3} \bullet \hat{\boldsymbol{\rho}}_{1}\right)}{\sqrt{1-\left(\hat{\boldsymbol{\rho}}_{3} \bullet \hat{\boldsymbol{\rho}}_{1}\right)^{2}}} \\
& =\frac{\hat{\boldsymbol{\rho}}_{3}-\hat{\boldsymbol{\rho}}_{1}\left(\hat{\boldsymbol{\rho}}_{3} \bullet \hat{\boldsymbol{\rho}}_{1}\right)}{\sqrt{1-\left(\hat{\boldsymbol{\rho}}_{3} \bullet \hat{\boldsymbol{\rho}}_{1}\right)^{2}}} \\
& \text { and } \\
& \hat{\boldsymbol{\xi}}=\hat{\boldsymbol{\xi}} \times \hat{\boldsymbol{\eta}}
\end{aligned}
$$

The next step is making a rotation matrix that will change the axis systems. The unit vectors in the direction of the observation become,

$$
\hat{\boldsymbol{\rho}}_{1}=\left[\begin{array}{l}
\lambda_{1} \\
\mu_{1} \\
v_{1}
\end{array}\right]=\left[\begin{array}{l}
1 \\
0 \\
0
\end{array}\right], \hat{\boldsymbol{\rho}}_{2}=\left[\begin{array}{l}
\lambda_{2} \\
\mu_{2} \\
v_{2}
\end{array}\right]=\left[\begin{array}{c}
\hat{\boldsymbol{\rho}}_{2} \bullet \hat{\xi} \\
\hat{\boldsymbol{\rho}}_{2} \bullet \hat{\boldsymbol{\eta}} \\
\hat{\boldsymbol{\rho}}_{2} \bullet \hat{\boldsymbol{\xi}}
\end{array}\right], \hat{\boldsymbol{\rho}}_{3}=\left[\begin{array}{c}
\lambda_{3} \\
\mu_{3} \\
v_{3}
\end{array}\right]=\left[\begin{array}{c}
\hat{\boldsymbol{\rho}}_{3} \bullet \hat{\xi} \\
\hat{\boldsymbol{\rho}}_{3} \bullet \hat{\boldsymbol{\eta}} \\
0
\end{array}\right]
$$


A first approximation to the constants $c_{1}$ and $c_{3}$ is found by only taking the first terms in the $f$ and $g$ series

$$
f=1 \text { and } g=t_{i}-t_{2}
$$

and by allowing

$$
\tau_{1}=\left(t_{1}-t_{2}\right), \tau_{2}=\left(t_{3}-t_{1}\right), \tau_{3}=\left(t_{3}-t_{2}\right)
$$

Substituting these values into equation (15) gives

$$
c_{1}=\frac{t_{3}-t_{2}}{1\left(t_{3}-t_{2}\right)-\left(t_{1}-t_{2}\right) 1}=\frac{\tau_{3}}{\tau_{3}-\tau_{1}}
$$

and

$$
c_{3}=-\frac{t_{1}-t_{2}}{1\left(t_{3}-t_{2}\right)-\left(t_{1}-t_{2}\right) 1}=-\frac{\tau_{1}}{\tau_{3}-\tau_{1}} .
$$

Next, the $\rho_{1}, \rho_{2}$, and $\rho_{3}$ components of equation (16) are derived from equation (19), which yields

$$
\begin{aligned}
& \rho_{2}=\frac{-c_{1} Z_{1}+Z_{2}-c_{3} Z_{3}}{v_{2}}, \\
& \rho_{3}=\frac{\rho_{2} \mu_{2}+c_{1} Y_{1}-Y_{2}+c_{3} Y_{3}}{c_{3} \mu_{3}}, \\
& \rho_{1}=\frac{\rho_{2} \lambda_{2}-c_{3} \rho_{3} \lambda_{3}+c_{1} X_{1}-X_{2}+c_{3} X_{3}}{c_{1}}
\end{aligned}
$$

where $\mathrm{X}_{i}, \mathrm{Y}_{i}$, and $\mathrm{Z}_{i}$ are the heliocentric positions of the Earth at time $i$. These three equations with three unknowns are solved producing $\rho_{1}, \rho_{2}$, and $\rho_{3}$. Once the magnitudes are found, $\mathbf{r}_{i}$ can be found by using equation (15). But $c_{1}$ and $c_{3}$ also depend on $\mathbf{r}_{i}$,

$$
\begin{aligned}
& c_{1}=\frac{\left|\mathbf{r}_{2} \times \mathbf{r}_{3}\right|}{\left|\mathbf{r}_{1} \times \mathbf{r}_{3}\right|} \\
& c_{3}=\frac{\left|\mathbf{r}_{1} \times \mathbf{r}_{2}\right|}{\left|\mathbf{r}_{1} \times \mathbf{r}_{3}\right|} .
\end{aligned}
$$


These are Gauss' sector triangle ratios. The constants, $c_{1}$ and $c_{3}$, are now solved iteratively until the values for $\mathbf{r}(\mathrm{t})$ converge. Finally, $\dot{\mathbf{r}}$ can be found by using equation (17).

\subsection{Determination of Orbital Elements}

Next, the values of $\mathbf{r}$ and $\dot{\mathbf{r}}$ are used to find the orbital elements (e.g., Danby 1995). Since Kepler's First Law states that all orbits are ellipses, the orbital elements are values used to describe an ellipse. Therefore, a combination of the properties of an ellipse, conservation of energy, and Kepler's Laws are used to determine the orbital elements. To find the semi-major axis (a) the equation

$$
\frac{1}{\mathrm{a}}=\frac{2}{\mathbf{r}}-\frac{\dot{\mathbf{r}}^{2}}{\mu}
$$

is used. Next, to find the eccentricity (e) two equations with two unknowns can easily be solved for,

$$
e \cos (E)=1-\frac{r}{a} \quad \text { and } \quad e \sin (E)=\frac{r \cdot \dot{r}}{\sqrt{a \mu}}
$$

The eccentric anomaly (E) is defined in Figure 10.

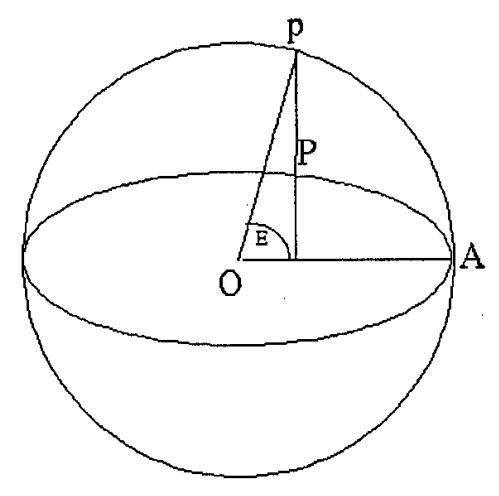

Fig. 10. -- Illustration of the definition of the eccentric anomaly (E). Every ellipse has an associated circle. In this figure, $\mathrm{p}$ is the corresponding point on the circle to the arbitrary point $\mathrm{P}$ on the ellipse. 
Now that both $\mathrm{e}$ and $\mathrm{E}$ are known, the mean anomaly $\left(\mathrm{M}_{0}\right)$ can be found using

$$
M_{0}=E-e \sin (E)
$$

The longitude of the ascending node, $\Omega$, the inclination angle, $i$, and the argument of perihelion, $\omega$, can be found from the $\mathrm{x}, \mathrm{y}$, and $\mathrm{z}$ components of $\mathbf{h}$,

$$
\begin{aligned}
& \mathbf{h}_{\mathrm{x}}=\mathbf{h} \sin (\Omega) \sin (i), \\
& \mathbf{h}_{\mathrm{y}}=\mathbf{h} \cos (\Omega) \sin (i), \\
& \mathbf{h}_{\mathrm{z}}=\mathbf{h} \cos (i)
\end{aligned}
$$

where $\mathbf{h}$ is the angular momentum per unit mass, $\mathbf{h}=\mathbf{r} \times \dot{\mathbf{r}}$, and is perpendicular to the orbital plane. Finally, the argument of perihelion $(\omega)$ needs to be found. The vector pointing towards the perihelion with an absolute value $\mathrm{e}$ is

$$
\mathbf{e}=\left(\mathrm{e}_{\mathrm{x}}, \mathrm{e}_{\mathrm{y}}, \mathrm{e}_{\mathrm{z}}\right)=\frac{\mathbf{v} \times \mathbf{h}}{\mu}-\hat{\mathbf{r}}
$$

Also, from geometry,

$$
\mathrm{e} \cos (\omega)=\mathrm{e}_{\mathrm{x}} \cos (\Omega)+\mathrm{e}_{\mathrm{y}} \sin (\Omega)
$$

and

$$
\mathrm{e} \sin (\omega)=\frac{\mathrm{e}_{\mathrm{z}}}{\sin (i)}
$$

By combining these three equations, the argument of perihelion ( $\omega)$ can be found. 


\subsection{Perturbed Motion}

Gauss' Method only takes the gravitational interaction with the Sun into account. However, other objects in the Solar System, such as Earth, may influence the orbit of the NEA. Perturbing forces are the gravitational forces exerted on the asteroid by bodies other than the Sun. These forces cause the orbit to change over time. There are two main methods that are used to account for these forces explicitly. The first is based on tidal perturbations in a binary system (such as the Earth-Moon system). It was first introduced by Newton and formalized by Lagrange as partial derivatives of the perturbing force with respect to the elements. The second method comes from Gauss. Gauss resolves the perturbing force into a coordinate system, which is rotating with the orbital motion (e.g., Danby 1988). However, these calculations are complicated, computer intensive and beyond the scope of this project, especially considering that it is fruitless to add perturbations to an orbit if more observations are needed to solidify the values of the orbital elements. On the other hand, the Minor Planet Center calculates orbits including perturbations where it is justified. The conclusions made here regarding the possibility of a close encounter ${ }^{22}$ with these NEAs do not differ significantly from those made by the Minor Planet Center.

${ }^{22} \mathrm{~A}$ close encounter is within $0.2 \mathrm{AU}$ of Earth. 


\section{$\underline{4 . ~ L i g h t c u r v e s}$}

A lightcurve is obtained by observing a source regularly throughout at least one full night from sunset to sunrise. As shown in Figure 25, a lightcurve is a graph of magnitude vs. time used to determine three physical parameters. First, a lightcurve can be used to determine the general shape of the asteroid. As the asteroid rotates, different sides face the Earth. Assuming the asteroid is not a sphere and that its rotational axis is not along our line of sight, as it rotates the reflected light from the Sun will vary. So by looking at the amplitude of magnitude variations, the shape of the asteroid is estimated.

The flux of light observed at the Earth (in $\frac{\mathrm{W}}{\mathrm{m}^{2}}$ ) is given by

$$
\mathrm{f}=\mathrm{L} \cdot \frac{1}{4 \mathrm{r}^{2} \pi} \operatorname{As}^{2} \pi \frac{1}{4 \rho^{2} \pi}
$$

where $L_{\circ}$ is the luminosity of the Sun (in W), A is the albedo ${ }^{23}$ of the asteroid, $r$ is the distance from the Sun to the asteroid, $\rho$ is the distance from the Earth to the asteroid, and $\mathrm{s}$ is the radius of the asteroid. As the asteroid rotates, the cross-sectional area facing the Earth changes, but all other parameters remain the same $\mathrm{e}^{24}$. So the maximum and minimum flux measured is related to the change in magnitude as

\footnotetext{
${ }^{23}$ Albedo is the fraction of incident light that is reflected.

${ }^{24}$ All terms in the two fluxes are common terms except for the asteroid's radius. The luminosity of the Sun is a constant. The distance of Earth from the Sun and the distance of the asteroid from the Sun can both be assumed to be constant since only a small amount of time passes from one observation to the next.
} 


$$
\begin{aligned}
\mathrm{m}_{\max } & -\mathrm{m}_{\min }=\frac{5}{2} \log \left(\frac{\mathrm{f}_{\min }}{\mathrm{f}_{\text {max }}}\right) \\
& =\frac{5}{2} \log \left(\frac{\mathrm{s}_{\text {min }}}{\mathrm{s}_{\text {max }}}\right)^{2} . \\
& =5 \log \left(\frac{\mathrm{s}_{\text {min }}}{\mathrm{s}_{\text {max }}}\right)
\end{aligned}
$$

Then solving for the ratio of the radii gives the relative shape of the asteroid,

$$
\frac{\mathrm{s}_{\min }}{\mathrm{s}_{\max }}=10^{\left(\frac{\mathrm{m}_{\max }-\mathrm{m}_{\min }}{5}\right)} .
$$

A lightcurve is also used to determine the rotational period of the asteroid, assuming that every other peak in the lightcurve is due to reflected sunlight off the same face of the asteroid. The rotational period is obtained by finding a pattern in the lightcurve that repeats several times. The time between the two patterns is the rotational period.

Lightcurves of an asteroid from at least three different times that the asteroid is at opposition $^{25}$ are needed in order to find an asteroid's pole axis (e.g., Kiss, Szabo, \& Sarneczky 1999). Since this would take several years, this project focused on finding the rotational period and general shape of an asteroid from its lightcurve without finding its pole axis. Both the rotational period and shape are important parameters if a plan is required to deflect the asteroid from impacting Earth.

\footnotetext{
${ }^{25}$ Opposition is the point in the object's orbit when Earth is between it and the Sun, making it the brightest that we see it.
} 


\section{Results and Discussion}

\section{$\underline{5.1 \text { Cooperation Process }}$}

Ideally, an asteroid should be observed on nearly a dozen nights spread out over three months in order to calculate an orbit reliable for decades. For a number of reasons, it is not possible for a single observatory to obtain enough data to do this alone.

A major problem almost every optical observatory faces is bad weather, such as rain, snow, or cloud cover. This severe weather obviously closes the observatory for the night, but even more mild conditions threaten observing. For example, a light haze will impede observing because the particles in the clouds scatter the light from astronomical sources. The asteroids are so faint that any blurring caused by scattered light makes them disappear into the ambient noise of the image. Another hazardous weather condition is the humidity. If the humidity is too high, then condensation can form on the CCD. Condensation on the CCD can cause a couple of problems. First, it scatters the light hitting the $\mathrm{CCD}$, causing the image to be blurred and faint if at all visible. Also, if condensation starts to form in the control box, then condensation on the wires can cause short circuits. Of course, even with ideal weather conditions, equipment failure is still a threat. During the course of this project, the CCD broke twice, the computers in the dome were replaced, and the telescope tracking failed. Also, if the asteroid happens to be moving away from Earth while it is being tracked, then it will get fainter. This may make it impossible to track the asteroid for the full three month period.

One of the reasons that the Minor Planet Center exists is because no single observatory can reliably obtain enough observations of an asteroid to determine the orbit. 
Therefore, whenever an observatory finds the position of an NEA, it reports this position into the Minor Planet Center. The Minor Planet Center then posts these coordinates on the world-wide web so that anyone may use them to calculate the best orbit possible. To illustrate why it is important to use as much data as possible, Table 1 gives three different sets of orbital elements for asteroid 2000 EB107. One orbit is calculated from data obtained solely at the U.S. Naval Academy, the second orbit is calculated only from data posted on the world-wide web by the Minor Planet Center, while the third orbit is calculated from both of these data sets.

TABLE 1

Orbital Elements for $2000 \mathrm{~EB} 107$

\begin{tabular}{|l|l|l|l|l|l|l|}
\hline Source & $\begin{array}{c}\mathrm{a} \\
(\mathrm{AU})\end{array}$ & $\mathrm{e}$ & $\begin{array}{c}i \\
(\mathrm{deg})\end{array}$ & $\begin{array}{c}\Omega \\
(\mathrm{deg})\end{array}$ & $\begin{array}{c}\omega \\
(\mathrm{deg})\end{array}$ & $\mathrm{M}_{0}$ \\
\hline USNA & 1.2 & 0.63 & 37 & 180 & 230 & 64 \\
\hline MPC & 3.1 & 0.59 & 26 & 180 & 43 & 350 \\
\hline USNA+MPC & 2.7 & 0.55 & 24 & 180 & 44 & 340 \\
\hline
\end{tabular}
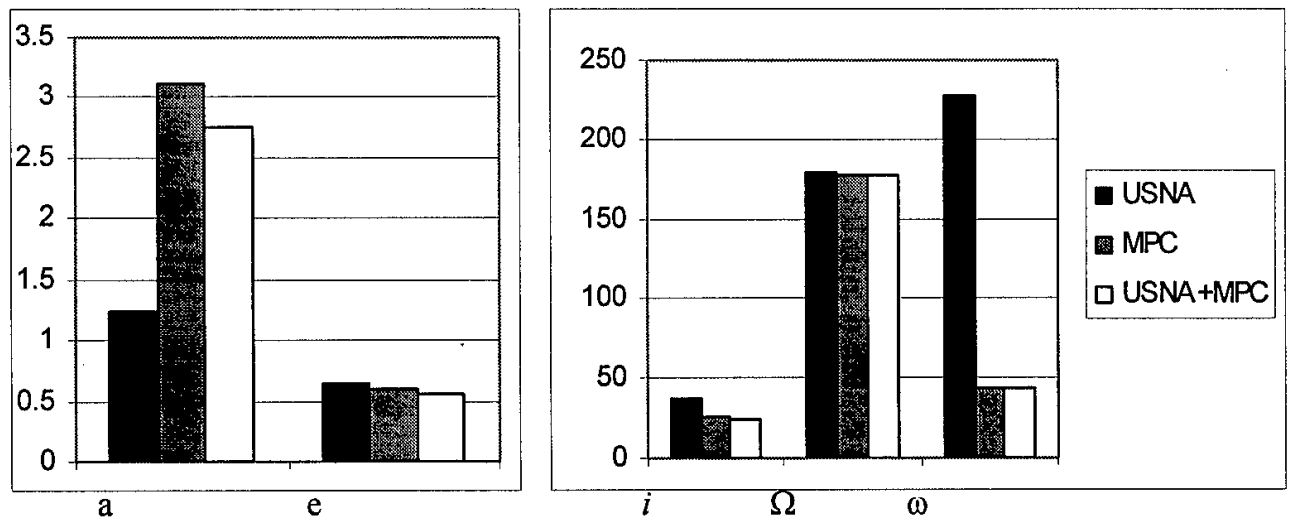

Fig. 11. -- Bar Graph comparing the orbital elements of 2000 EB107. 
As can be seen in Figure 11, there are great differences in the orbital elements, especially for the semi-major axis and the argument of perihelion. The semi-major axes differ by more than $1 \mathrm{AU}$, while the difference in the argument of perihelion is almost $200^{\circ}$. In Figure 12, these three orbits are shown. Even a quick inspection of Figure 12 clearly reveals that these differences in the orbital elements drastically change the shape of the orbit.

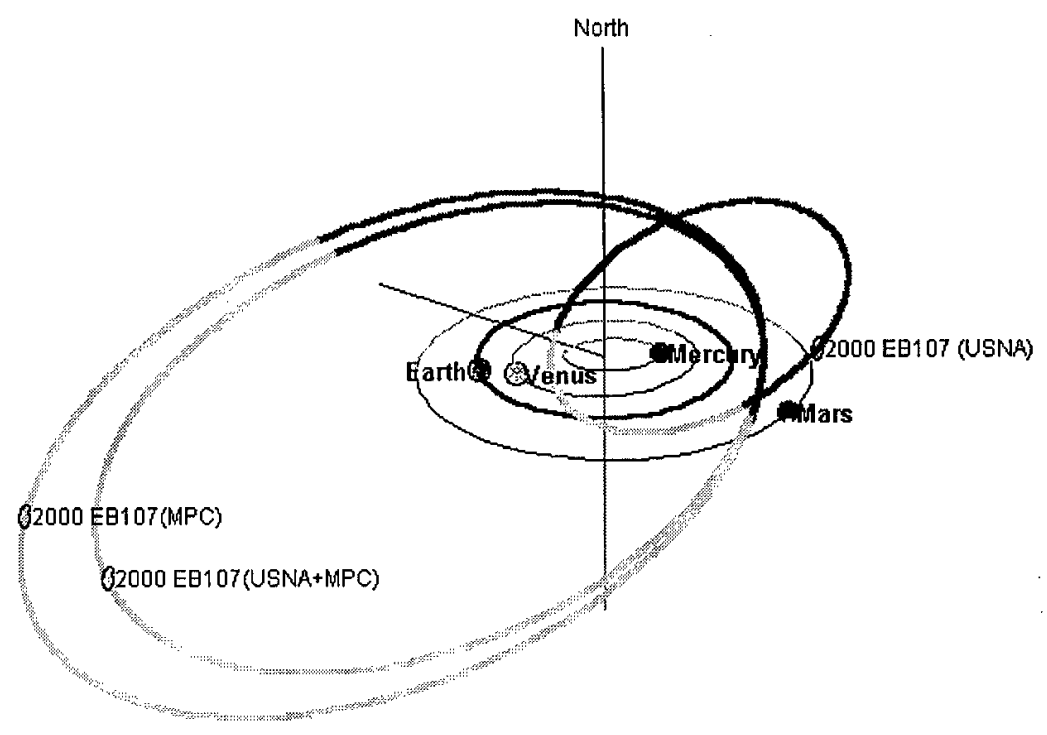

Fig. 12. -- The orbit of 2000 EB107 obtained by using data from different sources.

Because it uses the largest range of dates over which the asteroid was observed, the best orbit came from the combination of USNA and MPC data sets. 


\subsection{Using an Astronomical Calibrator}

The photometry software, Fitsblink, does not necessarily find the correct independent magnitude of the objects in an image. However, it does give the correct relative magnitude, meaning that the difference in magnitude of two objects in an image is correct. However, the difference in magnitude of an asteroid that is found between two images is not only due to an actual change in its magnitude, but also is due to an absolute calibration error between images. In order to correct for this, a reference star is used. This is simply a star that is found in the majority, if not all, of the images. In principle, the star's magnitude in each of these images should be the same, but it is not. To calibrate a series of images, all magnitudes are referred to an arbitrary observation of the reference star in one of the images. Figure 13 shows the lightcurve of 2000 EE104 with uncalibrated magnitudes and calibrated. In particular, points near 6 hours $\mathrm{UT}^{26}$ have changed by three magnitudes. This calibration greatly influences the interpretation of the data. As a final note, the main source of error in the magnitudes is due to the Guide Star Catalog. The Guide Star Catalog only reports magnitudes to within one third of a magnitude. This large error effectively drowns out any other source of error so that only this error need be addressed.

\footnotetext{
${ }^{26}$ UT is the universal time or the local standard time at the Prime Meridian.
} 
Fig. 13. -- Comparison of a raw and calibrated lightcurve.

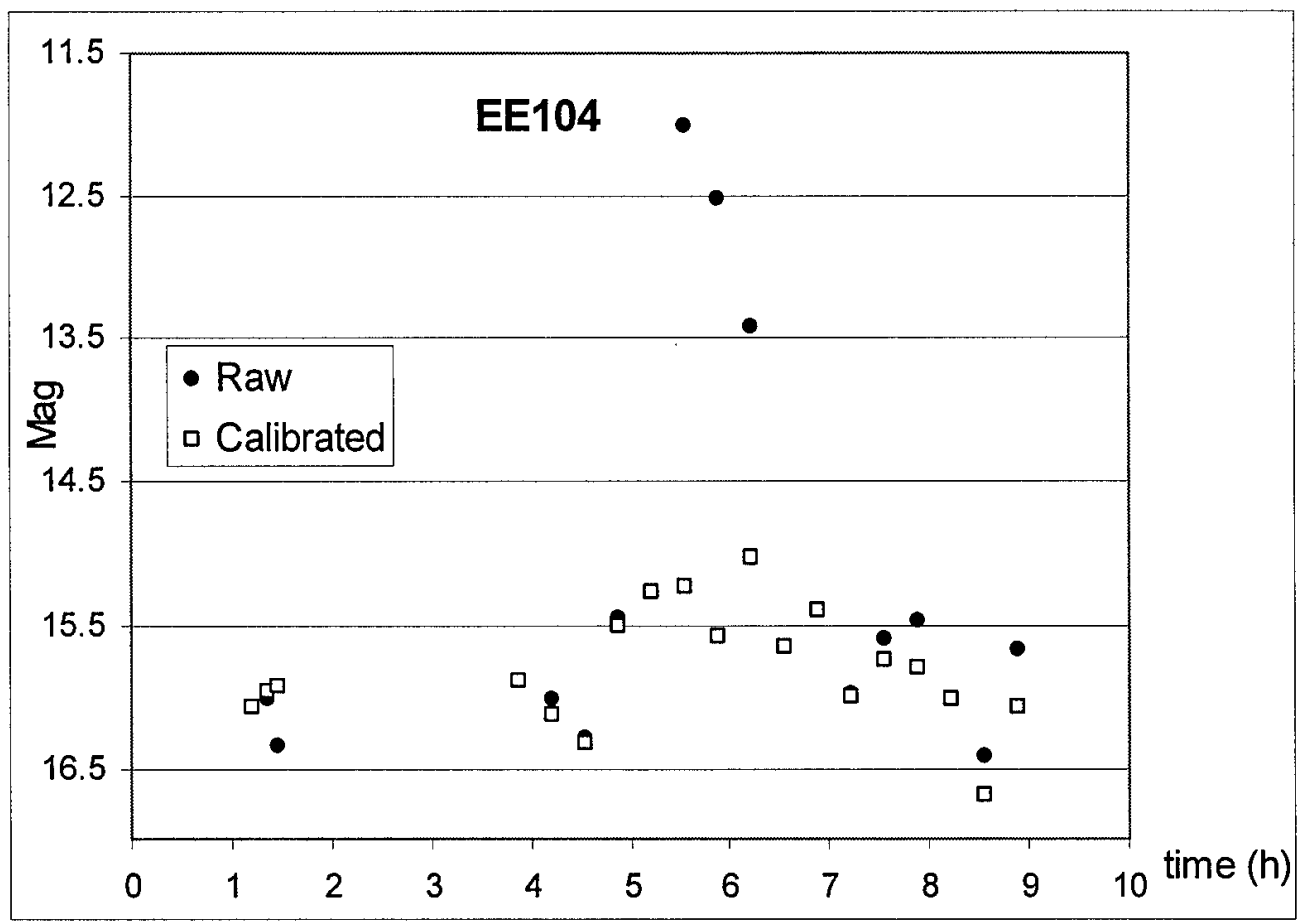

\subsection{Orbital Results and Discussion}

For this project, once enough observations were made to obtain an orbit, the orbital elements were found using a program provided by Spacewatch. This program uses five different methods to find the orbital elements and computes the $\mathrm{RMS}^{27}$ of the fits. The five methods are Gauss' (see Section 3.1), Circular, and three Viasala calculations. The Circular and three Viasala methods calculate the orbital elements assuming the eccentricity to be $0,0.15,0.30$, and 0.45 respectively. Circular or one of the three Viasala calculations is used when there is very little data, so the eccentricity must be assumed, and only the asteroid's position for the following night is required. The best fitting orbit has the lowest RMS value. Ideally, Gauss' Method is used because it does 
not assume any orbital elements. Table 2 displays the orbital elements that were found for each asteroid.

TABLE 2

Orbital Elements for the Asteroids that were Tracked

\begin{tabular}{|l|l|l|l|c|c|c|l|l|}
\hline Asteroid & $\begin{array}{l}\text { Calc. } \\
\text { Method }\end{array}$ & $\begin{array}{c}\mathrm{a} \\
(\mathrm{AU})\end{array}$ & $\mathrm{e}$ & $\begin{array}{c}i \\
(\mathrm{deg})\end{array}$ & $\begin{array}{c}\Omega \\
(\mathrm{deg})\end{array}$ & $\begin{array}{c}\omega \\
(\mathrm{deg})\end{array}$ & $\mathrm{M}_{0}$ & $\begin{array}{l}\text { Range of } \\
\text { Dates }\end{array}$ \\
\hline $\begin{array}{l}2000 \\
\text { DM8 }\end{array}$ & Gauss & 1.2 & 0.41 & 18 & 340 & 300 & 260 & 7 \\
\hline $\begin{array}{l}2000 \\
\text { DH8 }\end{array}$ & Gauss & 2.4 & 0.45 & 42 & 160 & 21 & 350 & 33 \\
\hline $\begin{array}{l}2000 \\
\text { DO1 }\end{array}$ & Viasala & 2.4 & 0.45 & 120 & 340 & 180 & 360 & 1 \\
\hline $\begin{array}{l}2000 \\
\text { EB107 }\end{array}$ & Gauss & 2.7 & 0.55 & 24 & 180 & 44 & 350 & 24 \\
\hline $\begin{array}{l}2000 \\
\text { DQ110 }\end{array}$ & Gauss & 3.3 & 0.63 & 58 & 350 & 95 & 20 & 13 \\
\hline $\begin{array}{l}2000 \\
\text { ED104 }\end{array}$ & Gauss & 1.3 & 0.28 & 31 & 190 & 230 & 110 & 23 \\
\hline $\begin{array}{l}2000 \\
\text { EE14 }\end{array}$ & Viasala & 1.3 & 0.15 & 98 & 180 & 43 & 4.3 & 27 \\
\hline $\begin{array}{l}2000 \\
\text { EB14 }\end{array}$ & Viasala & 1.5 & 0.15 & 99 & 160 & 2.8 & 350 & 4 \\
\hline $\begin{array}{l}2000 \\
\text { ED14 }\end{array}$ & Gauss & 0.87 & 0.23 & 4.4 & 5.5 & 310 & 220 & 4 \\
\hline $\begin{array}{l}2000 \\
\text { DP107 }\end{array}$ & Gauss & 1.4 & 0.37 & 8.4 & 360 & 290 & 260 & 31 \\
\hline $\begin{array}{l}2000 \\
\text { EE104 }\end{array}$ & Gauss & 0.98 & 0.18 & 3.1 & 27 & 280 & 260 & 22 \\
\hline
\end{tabular}

Note--As discussed in the text there are two sources of systematic error: gravitational perturbations and insufficient observations of the orbit. The random errors in the astrometry are approximately \pm 1 arcsec and $\pm 5 \mathrm{~s}$.

${ }^{27} \mathrm{RMS}$ is the root mean squared. A low root mean squared means the data lie close to the orbital fit and is therefore desirable. 


\section{Asteroid 2000 DM8}

Gauss' Method was used to determine the orbital elements for 2000 DM8. From the orbit it appears to be an Apollo asteroid. As can be seen in Figure 14, this initial calculation does not predict a close approach to Earth. At the places where it appears to cross Earth's orbit, it is far above Earth's orbit due to its inclination angle of $18.3^{\circ}$.

However, these conclusions are preliminary. Asteroid 2000 DM8 was lost after seven days of tracking because it became too faint for the equipment to detect.

Fig. 14. -- Orbit of 2000 DM8.

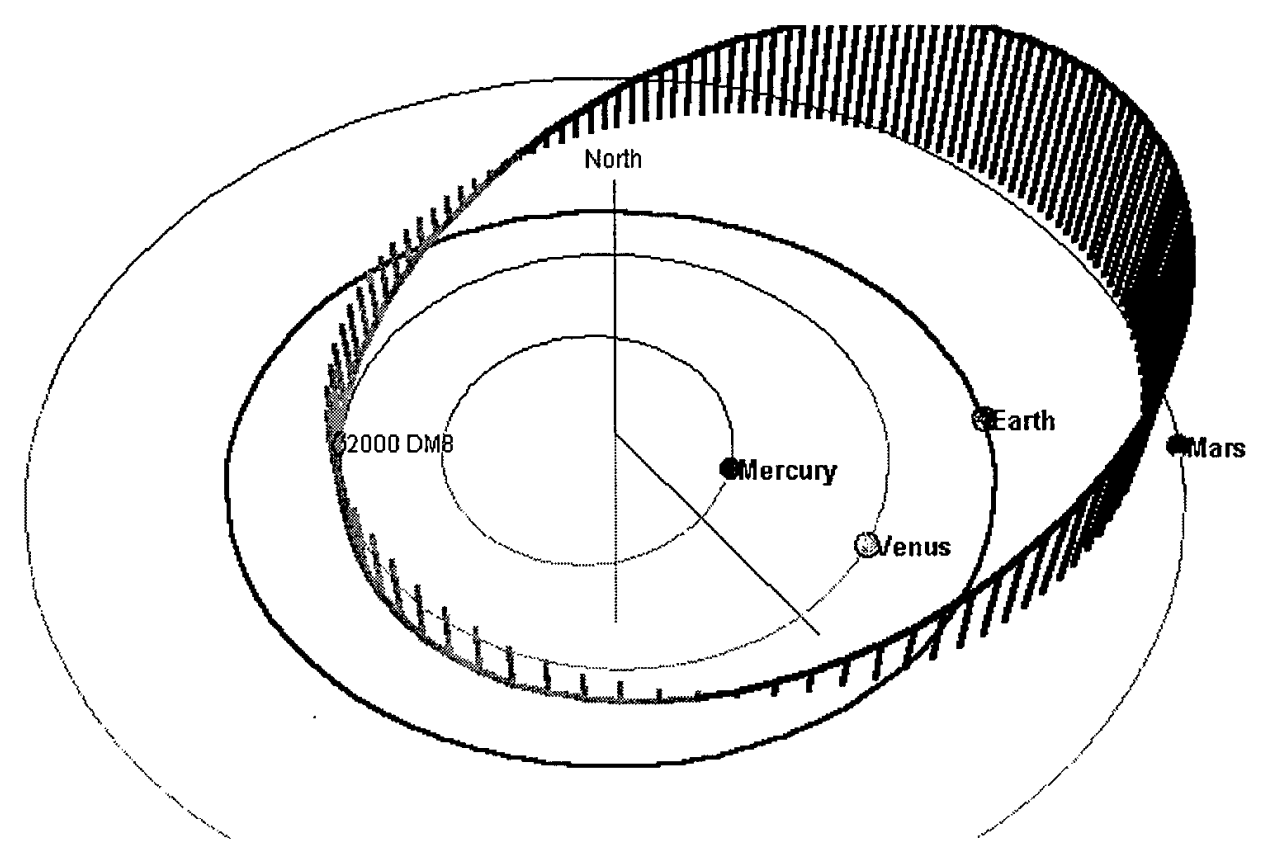




\section{Asteroid 2000 DH8}

The orbital elements of 2000 DH8 were also found using Gauss' Method. As can be seen in Figure 15, it does not appear to come close to the orbit of Earth. This orbit is reliable, since it was based on more than a month's worth of observations. Because this asteroid resides primarily in the main asteroid belt ${ }^{28}$ and is still bright enough to be easily seen, its size must be at least on the order of $50 \mathrm{~km}$. Therefore, it should continue to be tracked through May so that its orbit may be determined more accurately.

Fig. 15. -- Orbit of 2000 DH8.
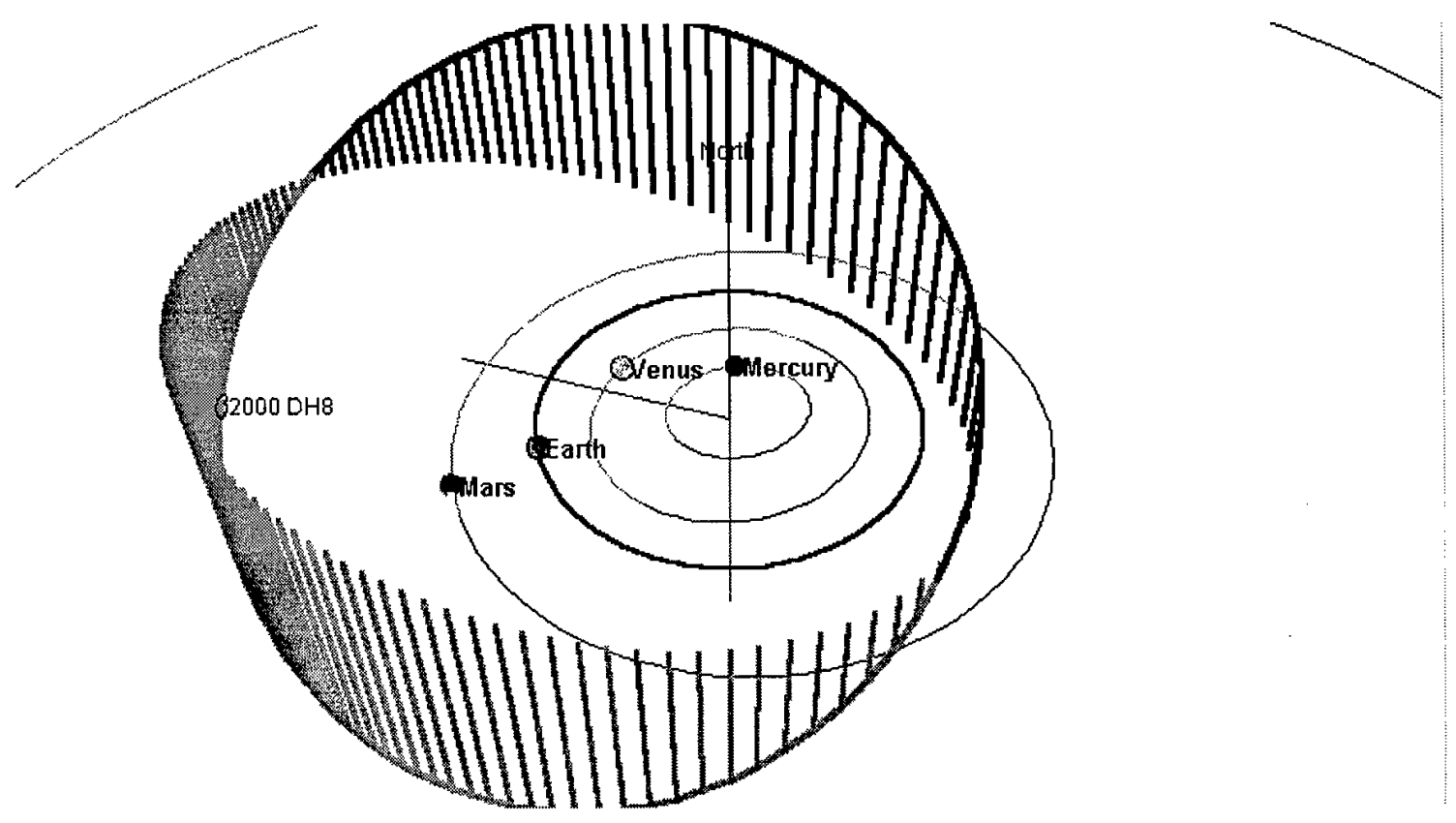

\footnotetext{
${ }^{28}$ The main asteroid belt is between the orbits of Jupiter and Mars and is where most asteroids can be found.
} 


\section{Asteroid 2000 DO1}

Figure 16 displays 2000 DO1's orbit. This orbit was based only on one night's

observation. Therefore, a Viasala calculation that assumed an eccentricity of 0.45

produced the best orbit. This orbit can only give the approximate position of the asteroid for a few nights; it cannot be used to determine if the asteroid may have a close approach with Earth. For these reasons, more observations need to be made in order to obtain a Gaussian orbit. However, it is likely to be lost because soon it will be too close to the Sun to observe.

Fig. 16. -- Orbit of $2000 \mathrm{DO}$.

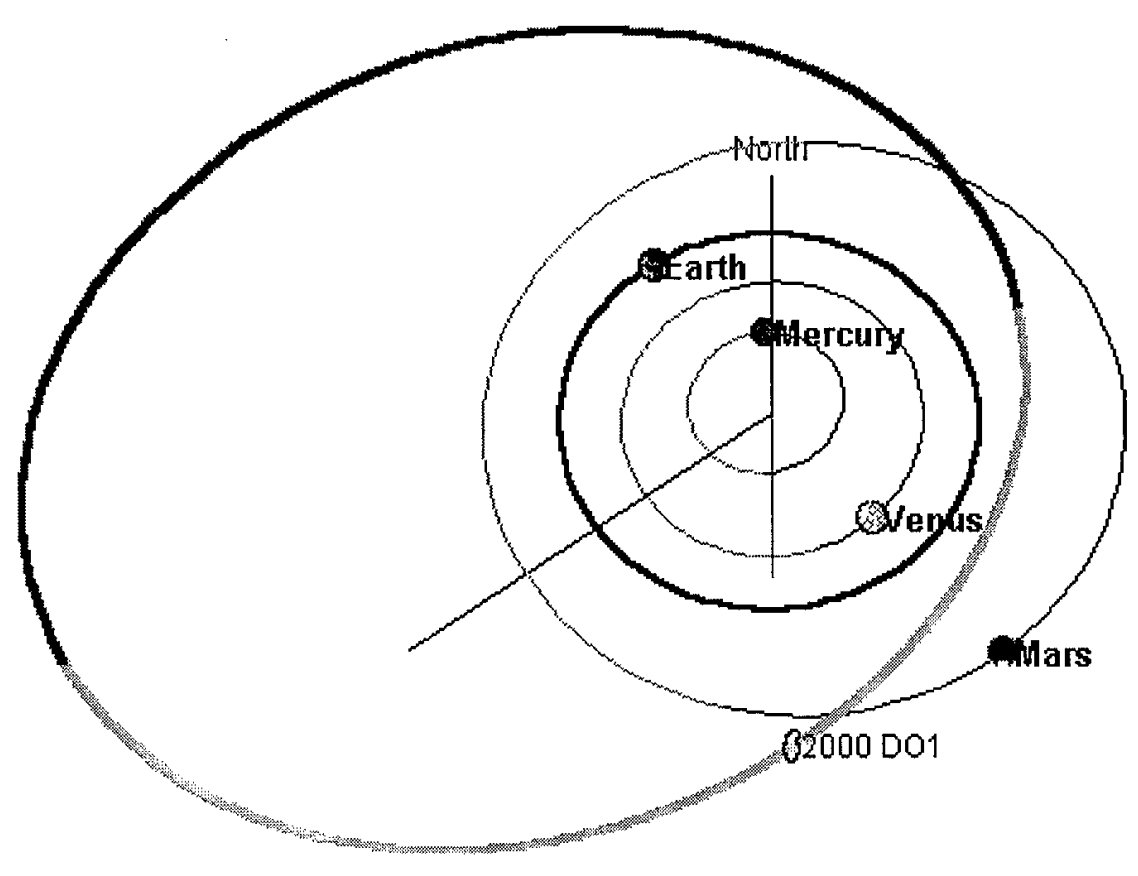




\section{Asteroid 2000 EB107}

A fairly well determined orbit for 2000 EB107 is displayed in Figure 17. It appears to be an Amor asteroid. From the figure, it seems that the asteroid will not collide with the Earth due to the fact that at the only place that its orbit comes within Mars' orbit, it is much farther out of the ecliptic with an inclination angle of $24^{\circ}$. Therefore, this asteroid is not a high priority for follow-up observations.

Fig. 17. -- Orbit of 2000 EB107.

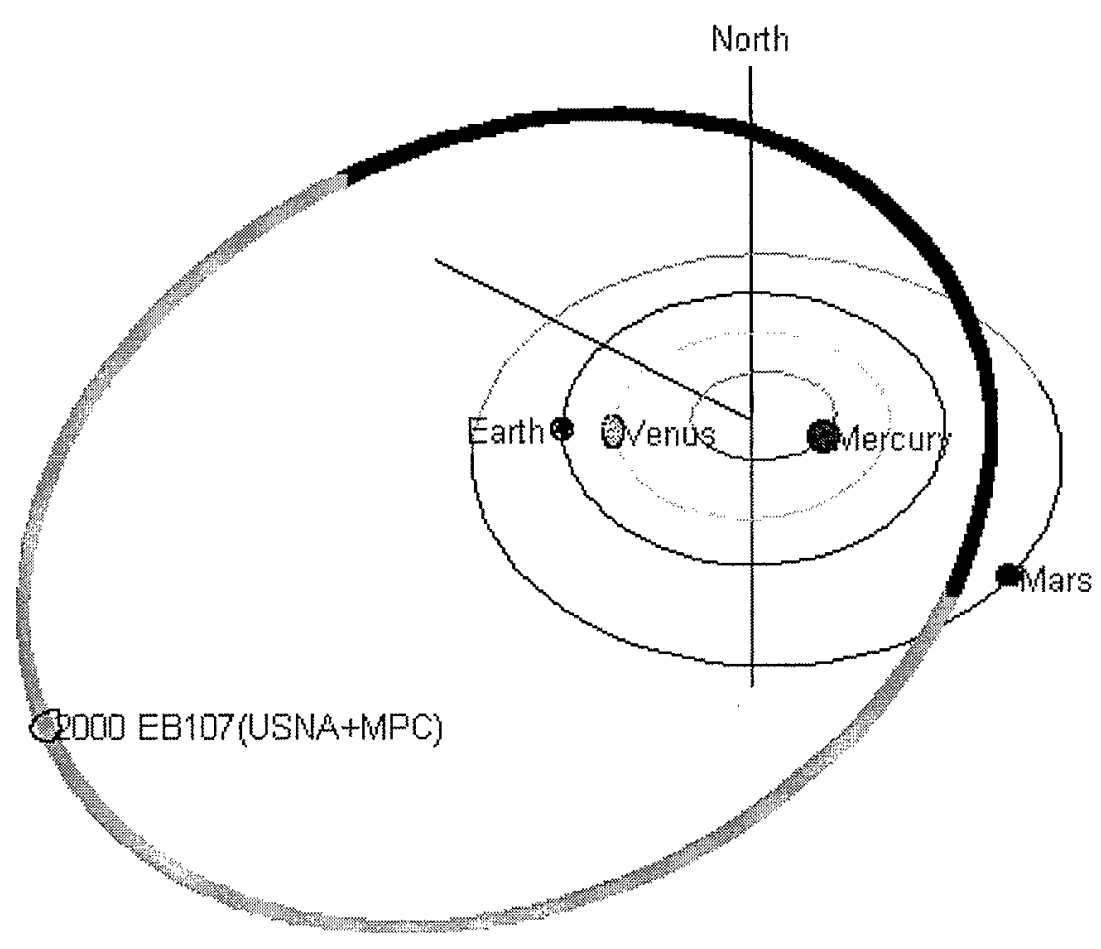


Despite the fact that the orbit of 2000 DQ110 was found by Gauss' Method, the most reliable method used, it is still suspect. Like $2000 \mathrm{DH} 8$, this asteroid appears to be a main belt asteroid, rather than an NEA (Figure 18). It has a large semi-major axis and eccentricity. Also, it was only observed over a period of thirteen days, requiring that further tracking be done to obtain a trustworthy orbit.

Fig. 18. -- Orbit of 2000 DQ110.

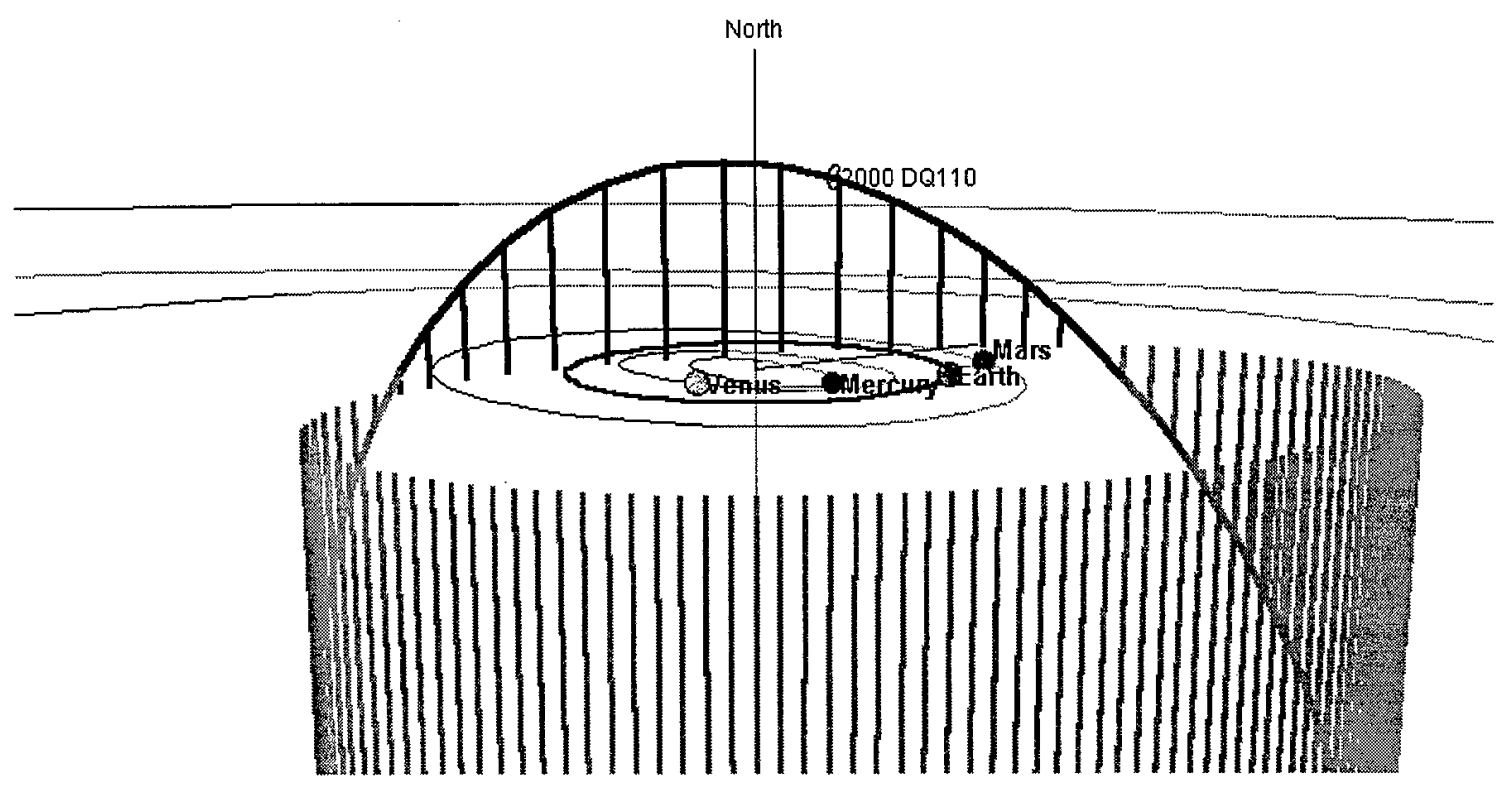


Asteroid 2000 ED104

With data spanning twenty-four days, a decent orbit of 2000 ED104 was

determined using Gauss' Method. Figure 19 suggests that 2000 ED104 is an Apollo asteroid and that it might have a close-approach with Earth. In fact, the Minor Planet Center predicts that it will be within 0.17AU of Earth on 9 October 2002.

Fig. 19. -- Orbit of 2000 ED104.

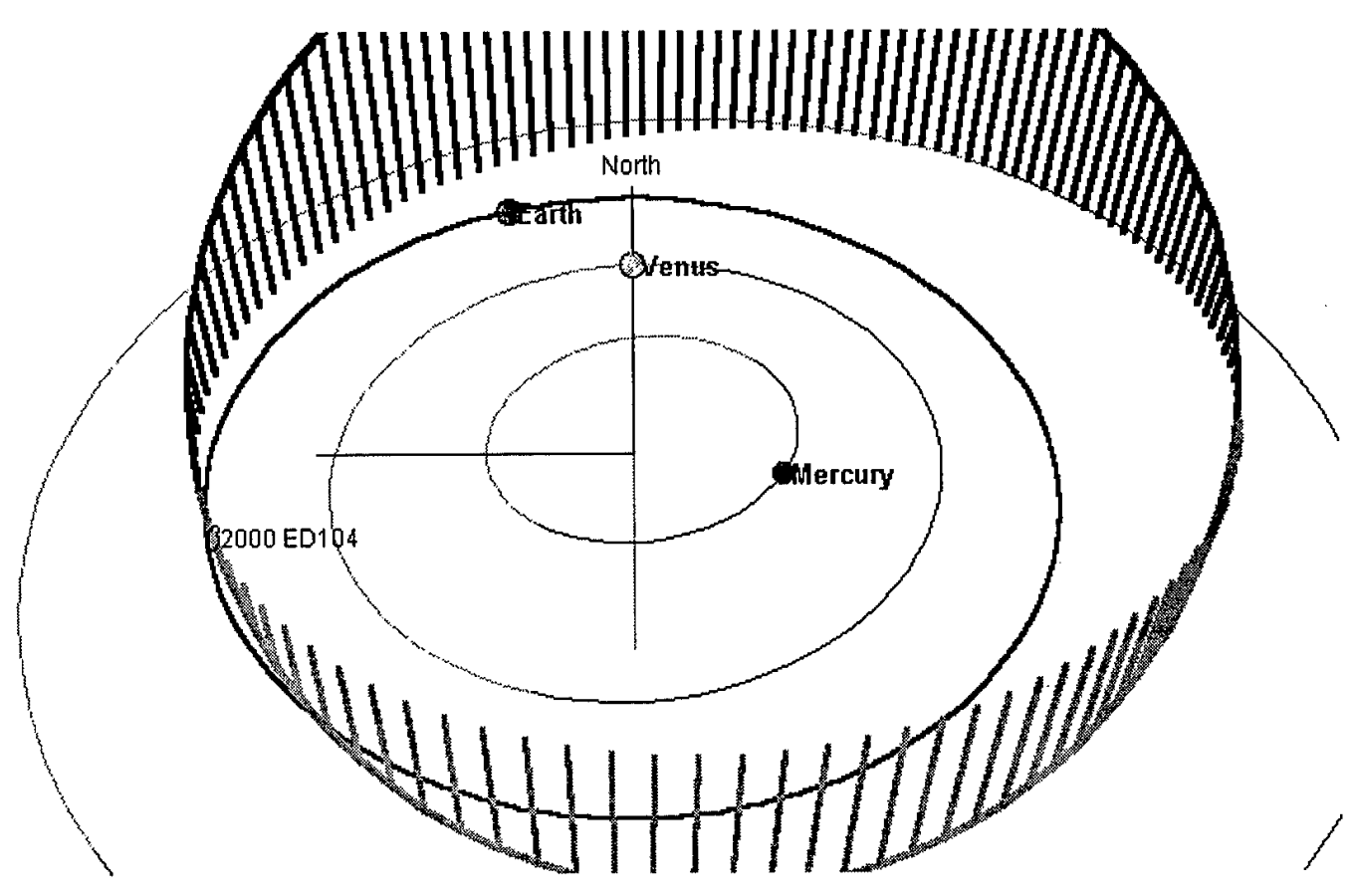


$\underline{\text { Asteroid } 2000 \text { EE14 and } 2000 \text { EB14 }}$

Although 2000 EE14 was observed over a time of twenty-seven days, a Gaussian solution could not be calculated due to the size of the gaps between observations. Asteroid 2000 EB14 was tracked for only four days. For both of these asteroids, the fact that a Viasala solution had to be used and their high inclination angles, as seen in Figures 20 and 21, make these asteroid's orbit untrustworthy. Both asteroids require more observations so that a Gaussian orbit can be calculated.

Fig. 20. -- Orbit of 2000 EE14.

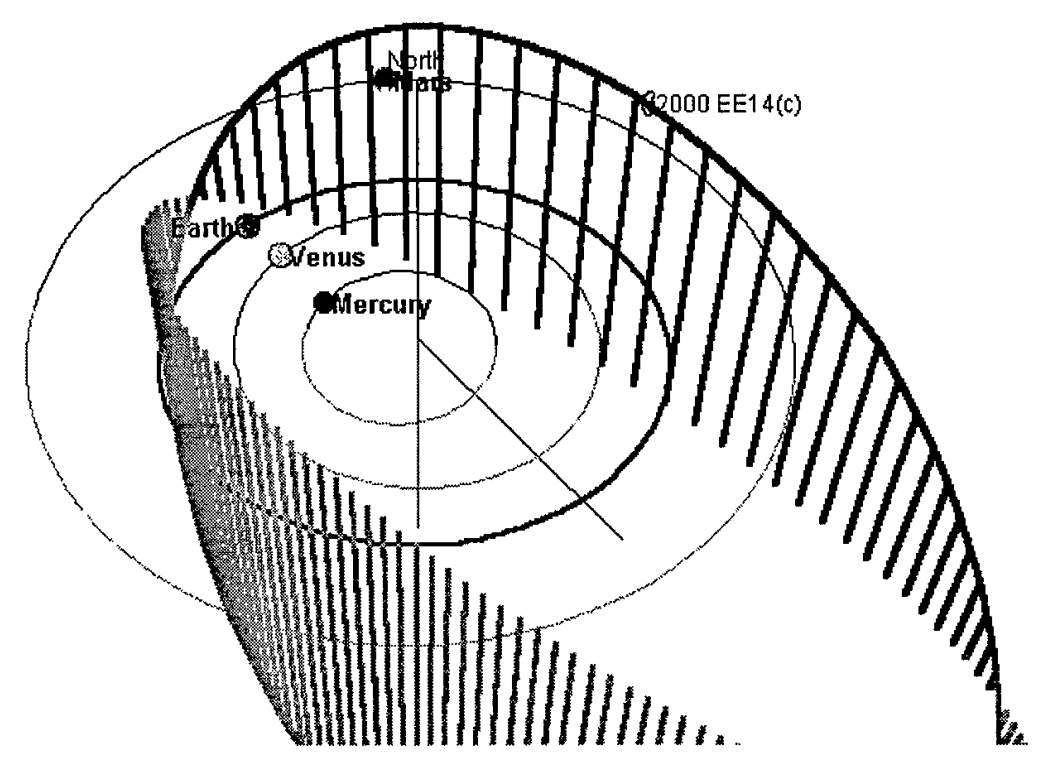


Fig. 21. -- Orbit of 2000 EB14.

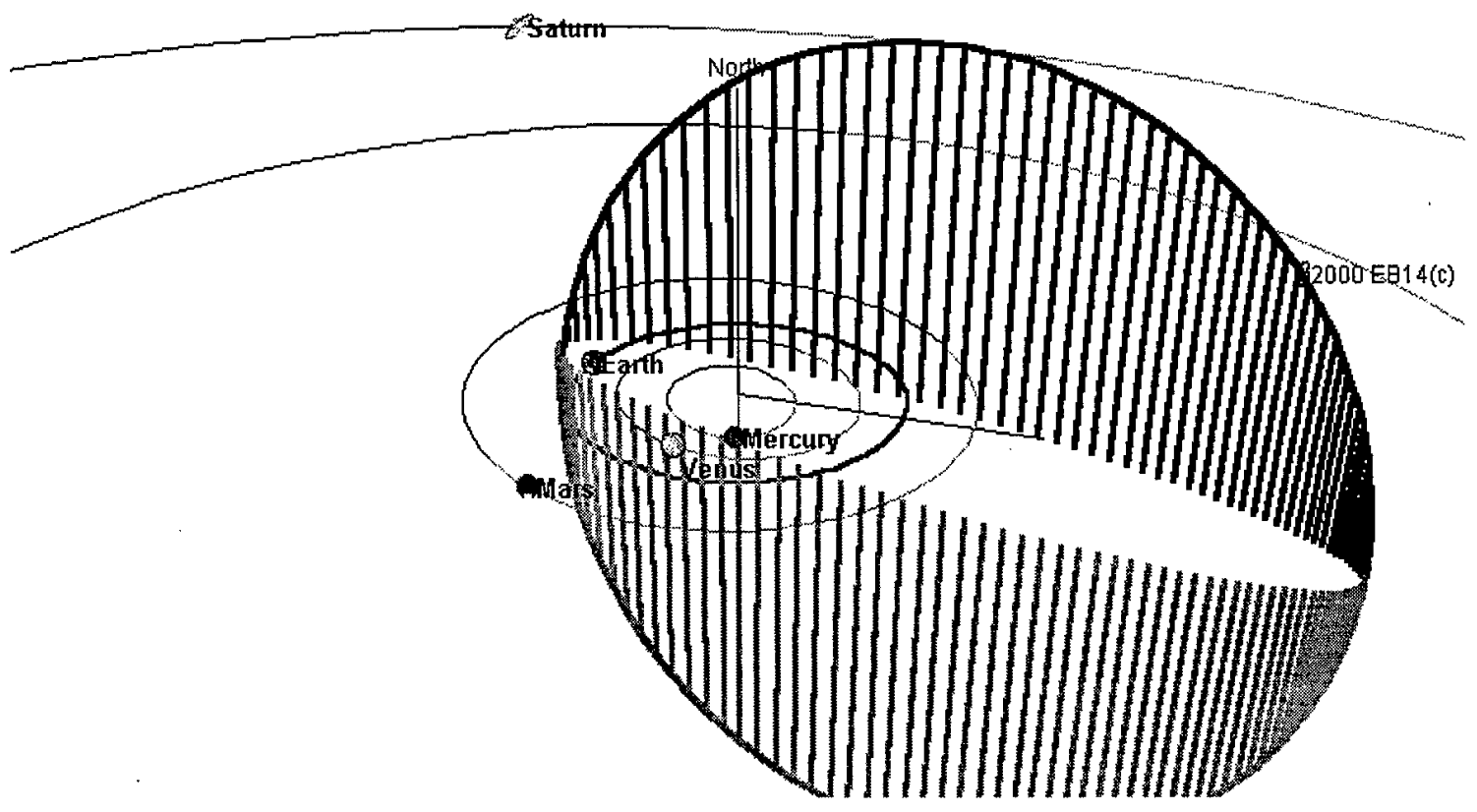


Asteroid 2000 ED14

Although 2000 ED14 has only been observed for a period of four days, its Gaussian orbit is tantalizing. Both the orbit shown in Figure 22 and the Minor Planet Center agree that it may have a close approach with Earth. In fact, according to the Minor Planet Center, this asteroid was merely 0.04AU from Earth, twice the Moon's distance from Earth, on March 16, 2000 (spring break), and it is predicted to come within 0.2AU of Earth in March 2013, March 2016, and March 2029. Clearly this asteroid is a top priority for further investigation.

Fig. 22. -- Orbit of 2000 ED14.

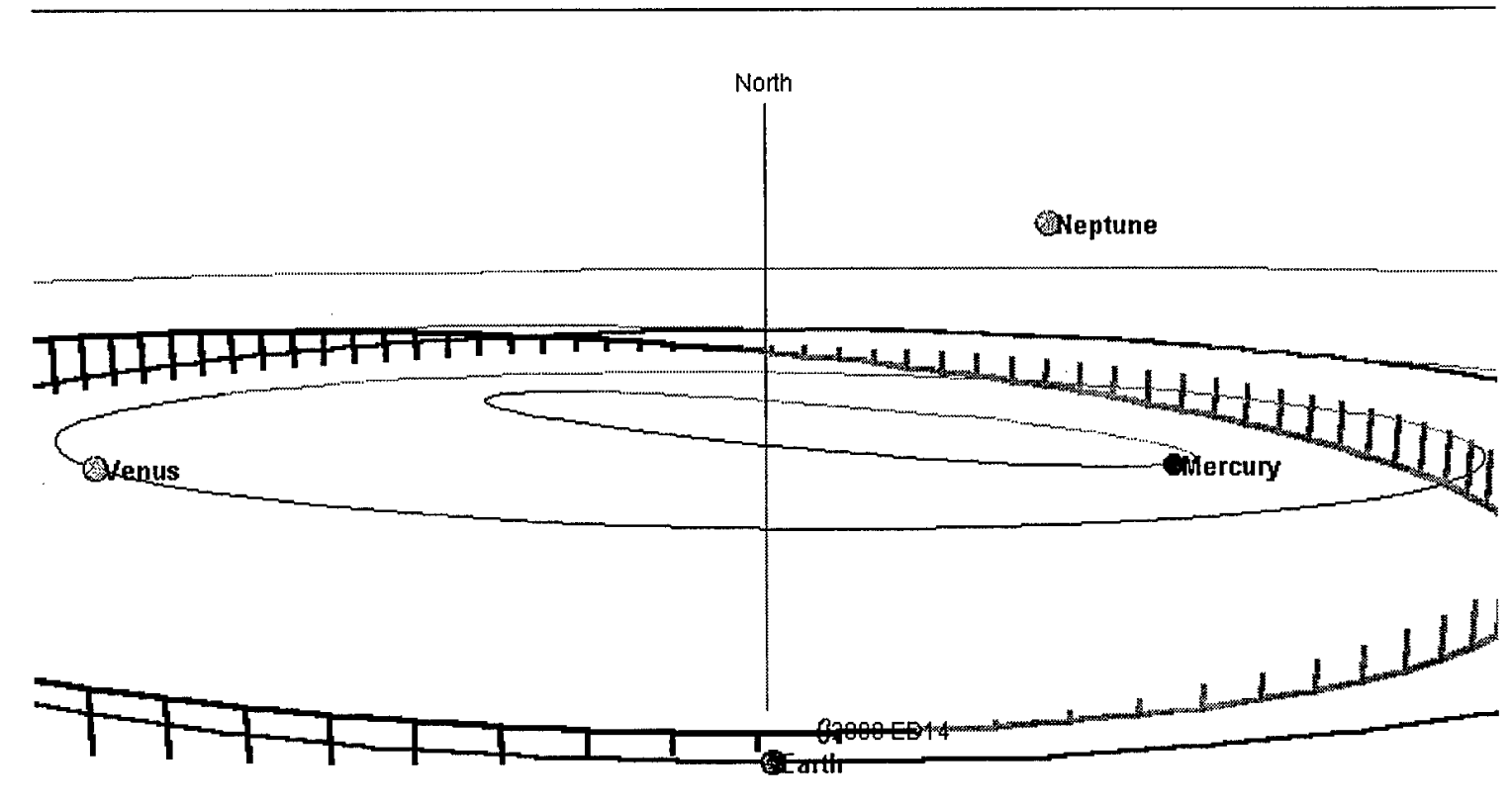


$\underline{\text { Asteroid } 2000 \text { DP107 }}$

This fairly well determined orbit, displayed in Figure 23, shows a close encounter with Earth. However, the Minor Planet Center does not report that this asteroid has a close approach with Earth. Due to this discrepancy and the fact that it may be a threat to Earth, further observations of 2000 DP107 is a top priority.

Fig. 23. -- Orbit of 2000 DP107.

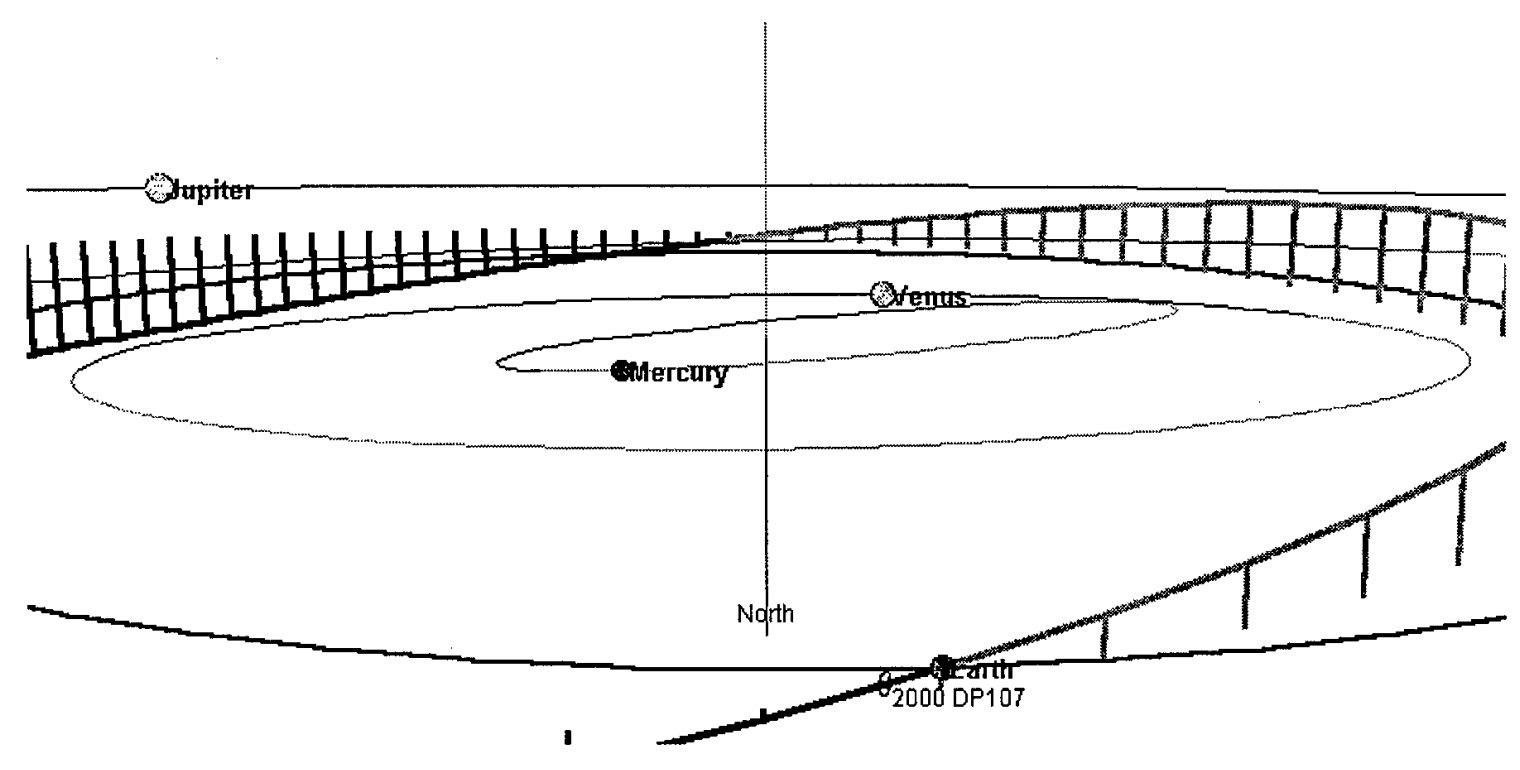


Asteroid $2000 \mathrm{EE} 104$

Both Figure 24 and the Minor Planet Center agree that this orbit will have a close approach with Earth. In fact, it was calculated that only six days before the due date of this project (11 April 2000) this asteroid was 0.04AU from Earth. It is predicted that this asteroid will have a close encounter with Earth twelve times in the next thirty-three years. Clearly this asteroid is a top priority for further investigation.

Fig. 24. -- Orbit of 2000 EE104.

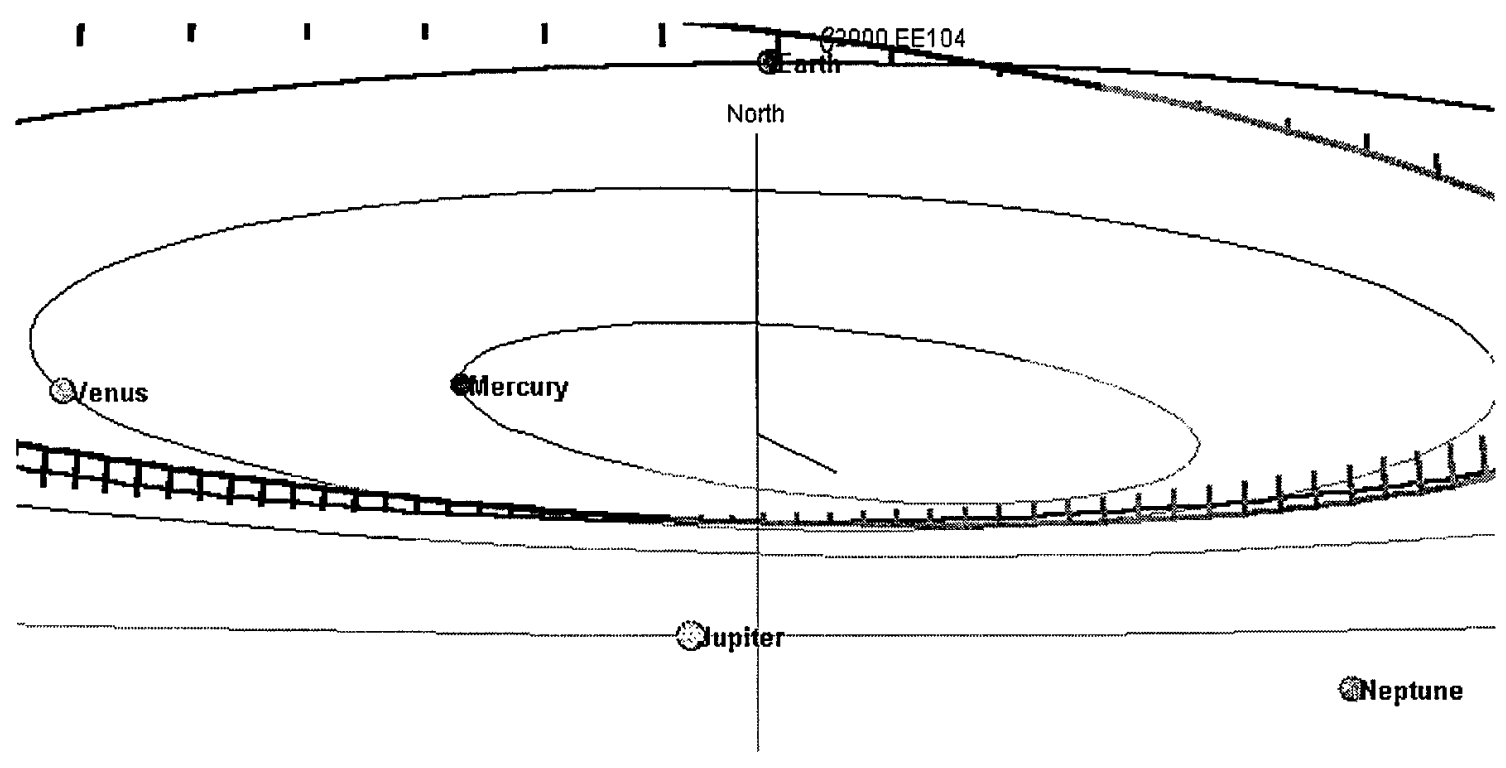


5.4 Lightcurve Results and Discussion

This section presents the lightcurves that were found for five of the eleven asteroids that were being tracked. Each lightcurve was observed over the period of one full night from sunset to sunrise. Two asteroids were observed for an additional night. On 1 April 2000 lightcurves were obtained for asteroids 2000 EB107 and 2000 EE104, while 2000 DM8 and 2000 DH8 were observed on 4 March and 6 March 2000. A partial lightcurve was observed for 2000 EE14 on 30 March 2000. Each image was then calibrated and the lightcurves obtained.

\section{Asteroids $2000 \mathrm{~EB} 107$ and $2000 \mathrm{EE} 104$}

The lightcurves for 2000 EB107 and 2000 EE104 are displayed in Figure 25. Except for a gap of two hours during which time other asteroids were being tracked, observations were made on each asteroid every twenty minutes from when the asteroid first rose to when it set. Due to these gaps in the data, only limits for the period can be found. The time from one maximum to the next is at most half of the period. Therefore the lower limits of the rotational period are 3.6 hours for 2000 EB107 and 8 hours for $2000 \mathrm{EE} 104$

For $2000 \mathrm{~EB} 107$, the brightest magnitude is 17.6 and faintest is 19.5 . The magnitude range for $2000 \mathrm{EE} 104$ is from 15.2 to 16.1. Using equation (34), $\frac{\mathrm{s}_{\max }}{\mathrm{s}_{\min }}=2.4$ for $2000 \mathrm{~EB} 107$ and $\frac{\mathrm{s}_{\max }}{\mathrm{s}_{\min }}=1.5$ for $2000 \mathrm{EE} 104$. These ratios are illustrated in Figure 27. 
Fig. 25. -- Lightcurves for 2000 EB107 and 2000EE104.
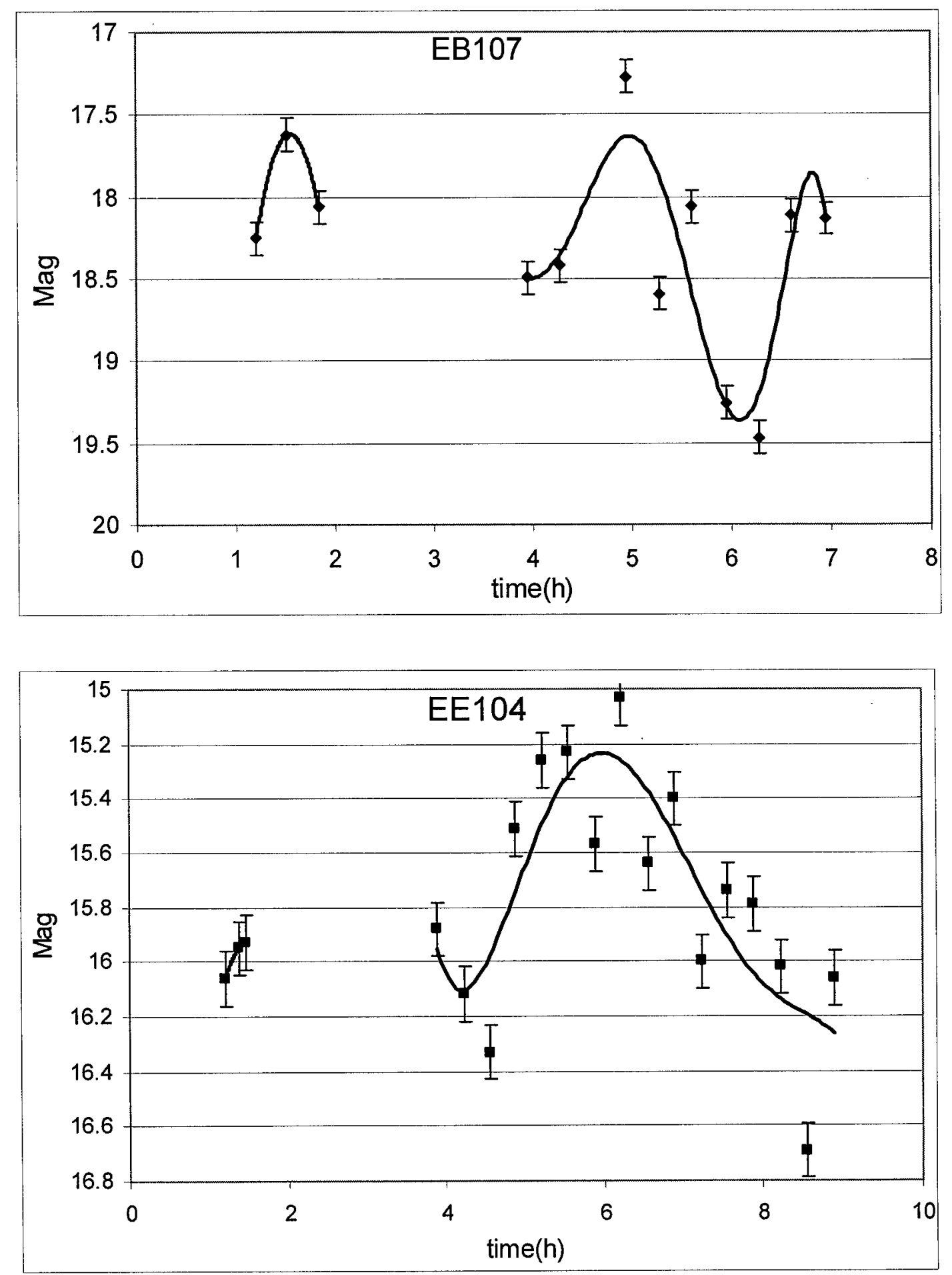
Asteroids 2000 DM8 and 2000 DH8

The data for these lightcurves, Figure 26, were taken over two nights. The rotational period of $2000 \mathrm{DM} 8$ is 4.6 hours. Because a clear pattern is not established in 2000 DH8 the rotational period can only be pinned down to a range between 3.2 and 5.5 hours. The brightest and faintest magnitudes for 2000 DM8 are 17.1 and 19.2. This gives $\frac{S_{\max }}{S_{\min }}=2.6$. The brightest and faintest magnitudes for 2000 DH8 are 17.6 and 19.0. This gives $\frac{\mathrm{s}_{\max }}{\mathrm{S}_{\min }}=1.9$. These ratios are represented by the shapes seen in Figure 27. Only about one and a half nights worth of data was used for these lightcurves due to the fact that the tracking on the telescope began to malfunction in the middle of the second night. Otherwise, these asteroids were observed every twenty minutes on both nights. Since the time of night on both nights do not correspond to the same phase of rotation of the asteroid, the data from the second night was shifted on the $\mathrm{x}$-axis so that it best fit the first night's data. 
Fig. 26. -- Lightcurves for 2000 DM8 and 2000 DH8.
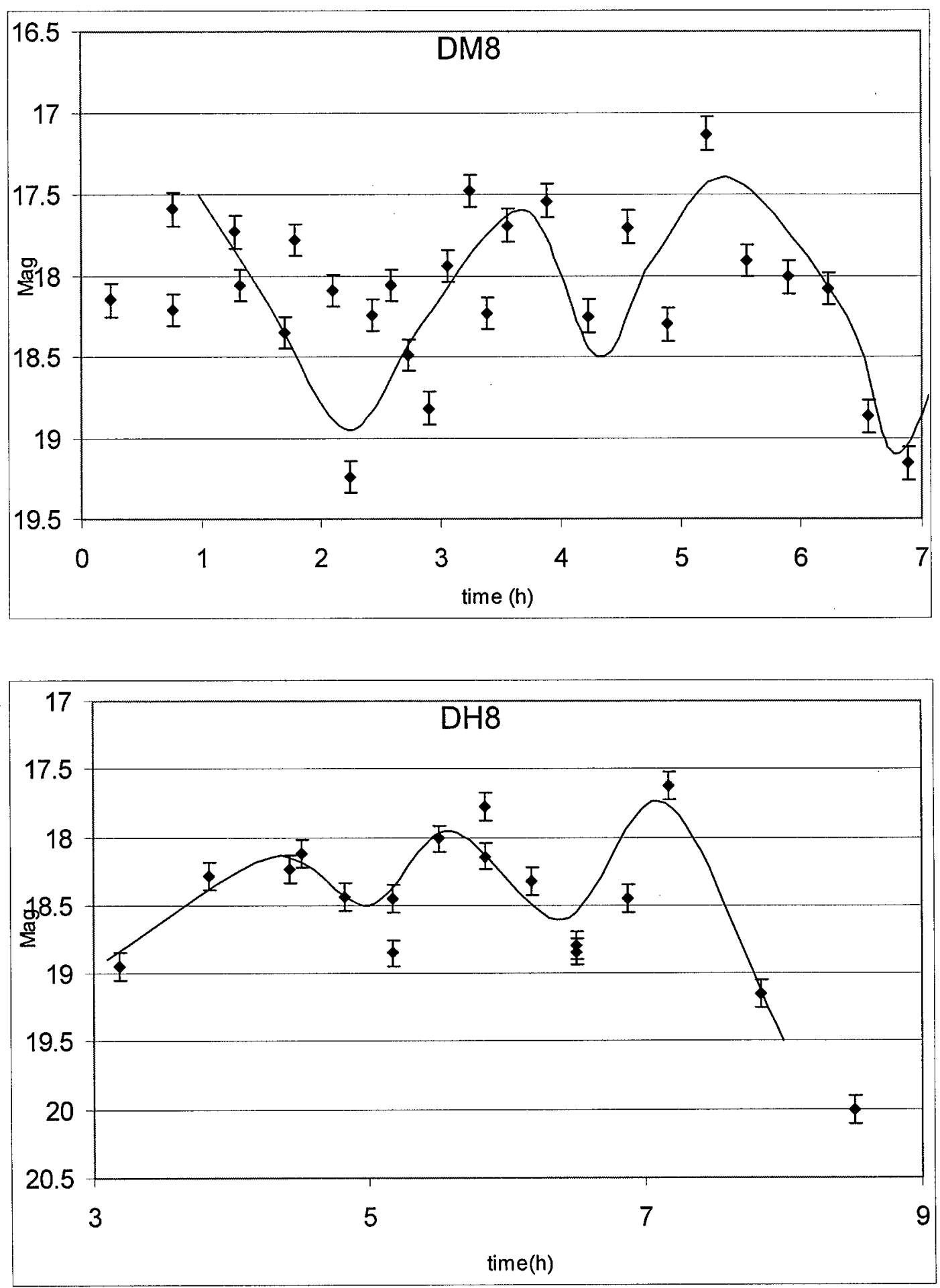
Fig. 27. -- Rough shapes based off radius ratios for $2000 \mathrm{~EB} 107,2000 \mathrm{EE} 104,2000 \mathrm{DH} 8$, and 2000 DM8.

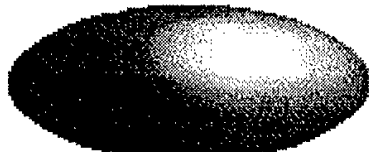

$2000 \mathrm{~EB} 107$

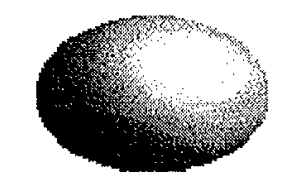

2000 EE104

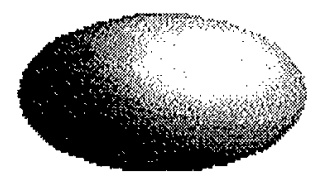

2000 DH8

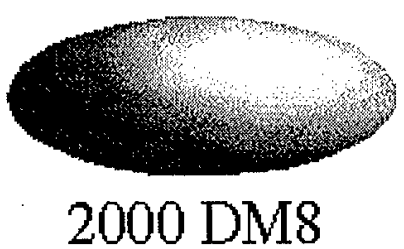

\section{Asteroid 2000 EE14}

The lightcurve for 2000 EE14 is shown in Figure 28. As can be seen from the plot, nothing conclusive can be determined. High humidity during the night caused condensation to form on the window of the CCD. Before this problem was discovered, 2000 EE14 was observed for approximately three hours. Most likely these data are corrupted by condensation. This plot is included to illustrate some of the observational problems that were encountered during this project and in hopes of helping others who may do similar observations at USNA. 
Fig. 28. -- Attempted lightcurve for 2000EE14.

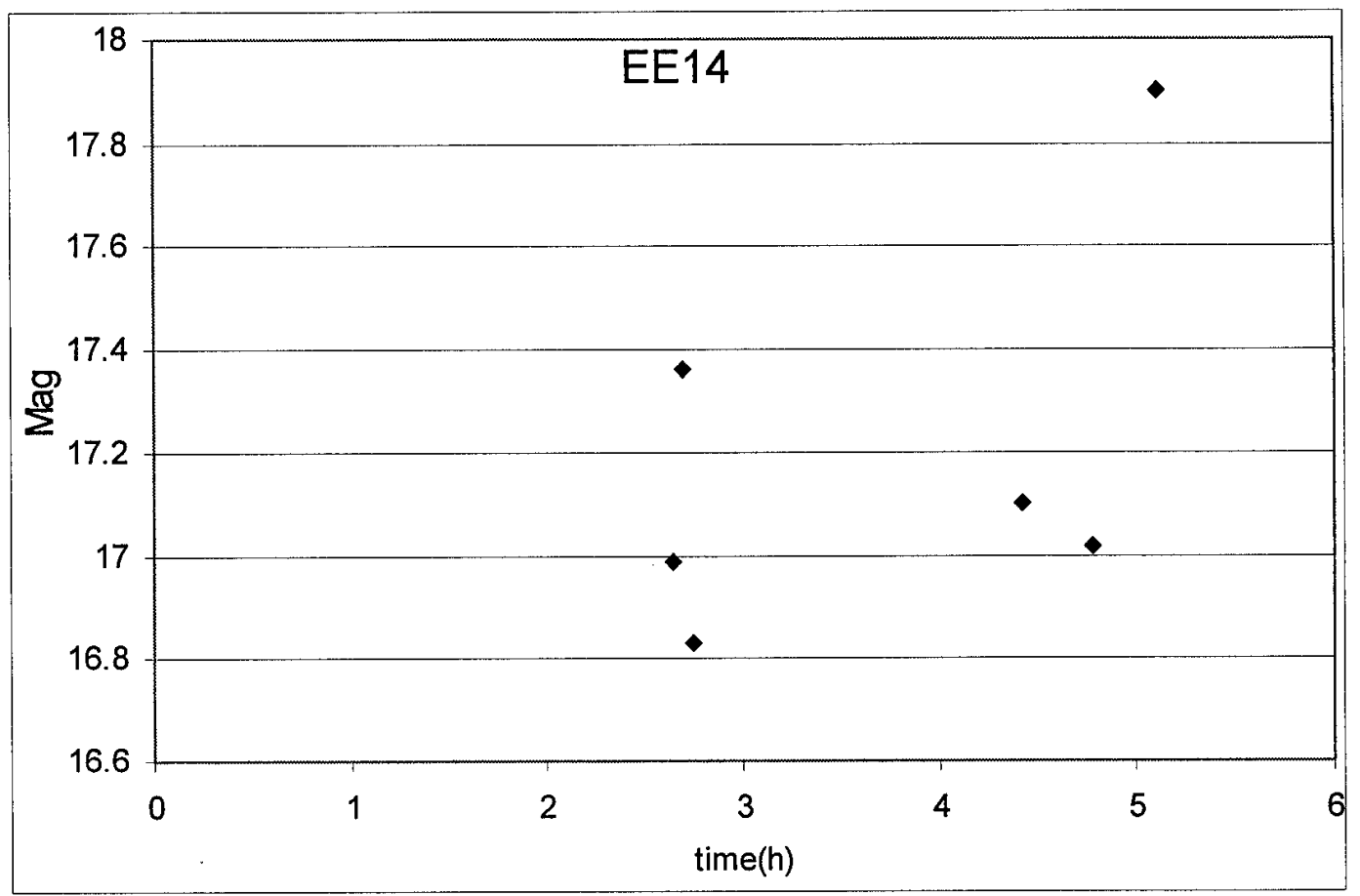


6. Conclusion

This project has emphasized the importance of carefully tracking NEAs. Of the eleven asteroids that were tracked for this project, approximately half will make a close approach to the Earth. Clearly these asteroids require further monitoring. But more importantly, the estimated 1500 undiscovered NEAs will need careful tracking.

Small observatories like the now fully operational one on the roof of Michelson Hall are ideally suited for such work. Many lessons have been learned as a result of this project. First, observing in the summer in Annapolis is nearly impossible due to the high humidity. Second, it is not useful to take lightcurves and track asteroids on the same night. This dual procedure leads to gaps in the lightcurve data which limit the analysis. Third, in order to compensate for degradation in tracking, the telescope must be reinitialized every one to two hours so that the desired object does not leave the CCD's field of view. Finally, same night analysis of observations must be made. This is so that any errors in the data can be seen early on and corrected, such as tracking, condensation, or focus. 


\section{Future Work}

For obvious reasons, this research is an ongoing project requiring more work.

First, the top priority asteroids, those with close approaches to Earth, will continue to be tracked so that a more definite orbit may be calculated. In addition, since the number of unknown NEA orbits is still large, observations of new asteroids will be done so that an orbit may be calculated for them. Not only will I do this over the next few months, but MIDN Aaron Fielder, USN will be doing this as part of an independent research project next semester. Also, more lightcurve data will be taken of 2000 EB107 and 2000 EE104 in order to fill in the existing gaps in their lightcurves. This is important because if MIDN Fielder then observed these same asteroids next year at opposition, only one more lightcurve would be needed to determine the pole axes of the asteroids. Another thing that will be done over the summer is a consolidation of the telescope workstation to a single, remote observing site on the third floor of Michelson Hall. Currently, the computer which controls the telescope is in one room, the computer which controls the $\mathrm{CCD}$ is on the roof of Michelson Hall, and the computer that analyzes the data is in a third room. The main obstacle to this consolidation will be finding a way to control the dome remotely and accurately. A remote site is important because the dust and weather conditions in the dome have already broken one monitor and keyboard. 


\section{REFERENCES}

Alvarez, L.W., Alvarez, W., Asaro, F., \& Michel, H.V. 1980, Science 208, 1095 Alvarez, L.W., \& Asaro, F. 1990, Scientific American 263, 78

Arny, T.T. 1998, Explorations: An Introduction to Astronomy. $2^{\text {nd }}$ Ed. (McGraw-Hill, Inc.: New York)

Binzel, R. P. 1989, "An Overview of the Asteroids." Asteroids II. (Tucson: University of Arizona Press)

Binzel, R. P., Farinella, P., Zappala, V., \& Cellino, A. 1989, "Asteroid Rotation Rates: Distributions and Statistics." Asteroids II. (Tucson: University of Arizona Press)

Boslough, M.B., Crawford, D.A., Trucano, T.G., \& Robinson, A.C. 1995, Geophys. Res. Lett., 22(13), 1821

Bowell, E., Chernykh, N.S., \& Marsden, B.G. 1989, "Discovery and Follow Up of Asteroids." Asteroids II. (Tucson: University of Arizona Press)

Chapman, C.R. \& Morrison, D. 1994, Nature 367, 33

Danby, J.M.A., Douzes, R., \& Whitney, C. 1995, Astrophysics Simulations The Consortium for Upper-level Physics Software (New York: John Wiley \& Sons, Inc.)

Danby, J.M.A. 1988, Fundamentals of Celestial Mechanics (Richmond: Willman-Bell, Inc.)

Fix, J.D. 1999, Astronomy: Journey to the Cosmic Frontier. (New York: McGraw-Hill, Inc.)

French, L. M., \& Binzel, R.P. 1989, "CCD Photometry of Asteroids." Asteroids II. (Tucson: University of Arizona Press)

Fujiwara, A., Cerroni, P., Davis, D.R., Ryan, E., Martino, M.D., Holsapple, K., \& Housen, K. 1989, "Experiments and Scaling Laws on Catastrophic Collisions." Asteroids II. (Tucson: University of Arizona Press)

Harris, A. W., \& Lupishko, D.F. 1989, "Photometric Lightcurve Observations and Reduction Techniques." Asteroids II. (Tucson: University of Arizona Press)

Kiss, L.L., Szabo, Gy., Sarneczky, K. 1999 Astronomy and Astrophysics Supplement 140,21

Martinez, P., \& Klotz, A. 1999, A Practical Guide To CCD Astronomy. Trans. Andre Demers. (Cambridge, United Kingdom: Cambridge University Press)

Morrison, D., Chapman, C., \& Slovic, P. 1994, In Hazards Due to Comets and Asteroids., ed. T. Gehrels (Tucson: University of Arizona Press), 59

Odewahn, S. C., Bryja, C., \& Humphreys, R.M. 1992, Astronomical Society of the Pacific, 104, 553

Rabinowitz, D. L. Astrophysical Journal, Part 1. 407, no. 1, 412

Shu, Frank H. 1982, The Physical Universe: An Introduction to Astronomy. (Mill Valley, California: University Science Books)

Taft, L.G. 1984, The Astronomical Journal, Vol. 89, Number 9, 1426

Toon, O.B., Zahnle, K., Turco, R.D., \& Corey, C. 1994, In Hazards Due to Comets and Asteroids, ed. T. Gehrels (Tucson: University of Arizona Press), 791

Vilas, F., Tholen, D.J., Lebofsky, L.A., Campins, H., Veeder, G.J., Binzel, R.P., \& Tokunaga, A.T. 1985, Icarus, 63, 201 
Zelik, M. \& Gregory, S.A. 1998, Introductory Astronomy and Astrophysics (Orlando: Saunders College Publishing) 


\section{Appendix A: Laplace's Method}

Laplace's Method for determining orbital elements uses four equations. Three of these equations are derived from the asteroid's geometrical relationship with Earth and the Sun, while the fourth represents the gravitational attraction between the Sun and the asteroid. The geometrical relationship between the asteroid, Earth, and the Sun can be seen in Figure 8. Summing the vectors, gives equation (15)

$$
\mathbf{R}+\mathbf{r}=\boldsymbol{\rho}=\rho \hat{\boldsymbol{\rho}}
$$

Taking the time derivative of equation (15) yields,

$$
\dot{\mathbf{R}}+\dot{\mathbf{r}}=\dot{\rho} \hat{\boldsymbol{\rho}}+\rho \dot{\hat{\boldsymbol{\rho}}}
$$

Taking the time derivative once more gives,

$$
\begin{aligned}
\ddot{\mathbf{R}}+\ddot{\mathbf{r}} & =\ddot{\rho} \hat{\boldsymbol{\rho}}+\dot{\rho} \dot{\hat{\boldsymbol{\rho}}}+\dot{\rho} \dot{\hat{\boldsymbol{\rho}}}+\rho \ddot{\hat{\boldsymbol{\rho}}} \\
& =\ddot{\rho} \hat{\boldsymbol{\rho}}+2 \dot{\rho} \dot{\hat{\boldsymbol{\rho}}}+\rho \ddot{\hat{\boldsymbol{\rho}}}
\end{aligned}
$$

The third equation relates the gravitational force between the bodies. Both the Earth and the asteroid have a gravitational attraction to the Sun, since the mass of the Sun is so much larger than the masses of the Earth or of the asteroid, the masses of the Earth and the asteroid are ignored. This relationship gives

$$
\ddot{\mathbf{r}}=-\mu \frac{\mathbf{r}}{\mathrm{r}^{3}} \quad \text { and } \quad \ddot{\mathbf{R}}=-\mu \frac{\mathbf{R}}{\mathrm{R}^{3}}
$$

where $\mu=\mathrm{GM}_{\text {sun }}$. If these two equations are summed and equation (15) is substituted for $\mathbf{r}$ then, 


$$
\begin{aligned}
\ddot{\mathbf{R}}+\ddot{\mathbf{r}} & =-\mu\left(\frac{\mathbf{R}}{\mathrm{R}^{3}}+\frac{\mathbf{r}}{\mathrm{r}^{3}}\right) \\
& =-\mu\left(\frac{\mathbf{R}}{\mathrm{R}^{3}}+\frac{\rho-\mathbf{R}}{\mathrm{r}^{3}}\right) .
\end{aligned}
$$

Now, combining equation (36) and (38) yields,

$$
-\frac{\mu \boldsymbol{\rho}}{\mathrm{r}^{3}}+\mu\left(\frac{1}{\mathrm{r}^{3}}-\frac{1}{\mathrm{R}^{3}}\right) \mathbf{R}=\ddot{\boldsymbol{\rho}} \hat{\boldsymbol{\rho}}+2 \dot{\boldsymbol{\rho}} \dot{\hat{\boldsymbol{\rho}}}+\rho \ddot{\hat{\mathbf{\rho}}}
$$

By using the Law of Cosines,

$$
r^{2}=\rho^{2}+R^{2}-2 R \rho \cos (\psi)
$$

is obtained. Therefore, the four equations needed for determining the orbital elements using Laplace's Method are equations (15), (35), (39), and (40).

Three observations of the asteroid give three values for $\hat{\boldsymbol{\rho}}$ and $\mathrm{t}$. From these observations, approximate values for $\dot{\hat{\boldsymbol{\rho}}}$ and $\ddot{\hat{\boldsymbol{\rho}}}$ are estimated. The initial approximation used in Laplace's method involves finding values for $\dot{\hat{\boldsymbol{\rho}}}$ and $\ddot{\hat{\boldsymbol{\rho}}}$ at some point in time. If this point in time is assumed to be $t_{0}$, then for small $t, \hat{\boldsymbol{\rho}}$ can be expanded as a Taylor series,

$$
\hat{\boldsymbol{\rho}}=(\hat{\boldsymbol{\rho}})_{\mathrm{t}=0}+\mathrm{t}(\dot{\hat{\boldsymbol{\rho}}})_{0}+\frac{1}{2} \mathrm{t}^{2}(\ddot{\hat{\boldsymbol{\rho}}})_{0}+\ldots
$$

By stopping this series after the third term, only three observations are necessary to find $(\dot{\hat{\mathbf{\rho}}})_{0}$ and $(\ddot{\hat{\mathbf{p}}})_{0}$. It is best to take $\mathrm{t}_{0}$ as either the arithmetic mean of the times or, if there are only three observations, as the central time. 
The next two steps are taken in order to reduce the number of unknown

variables. First, the dot product of equation (39) is taken with $(\hat{\boldsymbol{\rho}} \times \dot{\hat{\boldsymbol{p}}})$, so that

$$
\left(\frac{1}{\mathrm{r}^{3}}-\frac{1}{\mathrm{R}^{3}}\right)(\hat{\boldsymbol{\rho}} \times \dot{\hat{\boldsymbol{\rho}}}) \bullet \mathbf{R}=\rho(\hat{\boldsymbol{\rho}} \times \dot{\hat{\boldsymbol{\rho}}}) \bullet \ddot{\hat{\boldsymbol{\rho}}}
$$

Next, the dot product of equation (39) is taken with $(\hat{\boldsymbol{\rho}} \times \ddot{\hat{\mathbf{\rho}}})$,

$$
\left(\frac{1}{\mathrm{r}^{3}}-\frac{1}{\mathrm{R}^{3}}\right)(\hat{\boldsymbol{\rho}} \times \ddot{\hat{\boldsymbol{\rho}}}) \cdot \mathbf{R}=-2 \dot{\boldsymbol{\rho}}(\hat{\boldsymbol{\rho}} \times \dot{\hat{\boldsymbol{\rho}}}) \bullet \ddot{\hat{\boldsymbol{\rho}}}
$$

Once this is done, equation (40) and equation (42) can be solved for $r$ and $\rho$, equation (43) can then be solved for $\dot{\rho}$, and equation (15) and equation (35) can be solved for $\mathbf{r}$ and $\dot{\mathbf{r}}$, from which the elements of the orbit can be calculated. ${ }^{29}$

${ }^{29}$ Solving for the final values of $\mathbf{r}$ and $\dot{\mathbf{r}}$ is a complicated process involving a transcendental equation. 
